H. Naitoh

Nagoya Math. J.

Vol. 91 (1983), 119-149

\title{
PARALLEL SUBMANIFOLDS OF COMPLEX SPACE FORMS II
}

\author{
HIROO NAITOH
}

\section{Introduction}

This is a continuation of Part I, which appeared in this journal.

In the previous paper I we have defined the following notions: orthogonal Jordan triple system (OJTS), orthogonal symmetric graded Lie algebra (OSGLA), orthogonal Jordan algebra (OJA), Hermitian symmetric graded Lie algebra (HSGLA). And we have shown that equivalent classes of OJTS naturally correspond to equivalent classes of OSGLA and through this correspondence we have naturally constructed HSGLA's from the OJTS's associated with OJA's with unity.

In the present paper we will give OJA's with unity and thus HSGLA's associated with $r$-dimensional complete totally real parallel submanifolds of an $r$-dimensional complex space form of nonzero constant holomorphic sectional curvature. And we will show that rigid classes of these submanifolds correspond to equivalent classes of the associated OJA's with unity and thus the associated HSGLA's (Theorem 6.3).

Moreover, in the section 7, we will decompose these OJA's and HSGLA's into indecomposable ones (Theorem 7.10). The indecomposable OJA's and HSGLA's are devided into the following types: almost nilpotent type or simple type. In the section 8 we will determine almost nilpotent HSGLA's by using results of Cahen-Parker [2], and in the section 9 simple HSGLA's by using results of Berger [1] and Kobayashi-Nagano [6].

We retain the definitions and notations in Part $\mathrm{I}$.

\section{§ 6. The OJA and HSGLA associated with a complete inverse}

Let $L^{\ell}$ be an $\ell$-dimensional pseudo-Euclidean space and $N^{n}$ an $n$ dimensional connected parallel pseudo-riemannian submanifold of $\boldsymbol{L}^{\ell}$. Denote by $\tilde{\sigma}, \tilde{A}$ the second fundamental form, the shape operator of the

Received February 12, 1982. 
inclusion $N \hookrightarrow L^{\ell}$ respectively. The pseudo-riemannian metrics on $L^{\ell}$, $N$ will be denoted by the same notation \langle\rangle$_{L}$. Denote by $\hat{R}$ the curvature tensor for the Levi-Civita connection $\hat{\nabla}$ of $N$ and by $\tilde{R}^{\perp}$ the curvature tensor for the normal connection $\tilde{D}$ of the inclusion $N \hookrightarrow L^{\ell}$ respectively. Then we have the following identities:

$$
\begin{aligned}
& \hat{R}(X, Y) Z=\tilde{A}_{\tilde{\sigma}(Y, Z)} X-\tilde{A}_{\tilde{\sigma}(X, Z)} Y, \\
& \hat{R}(X, Y) \cdot \hat{R}=0, \\
& \tilde{R}^{\perp}(X, Y) \tilde{\sigma}(Z, W)=\tilde{\sigma}(\hat{R}(X, Y) Z, W)+\tilde{\sigma}(Z, \hat{R}(X, Y) W)
\end{aligned}
$$

for vector fields $X, Y, Z, W$ of $N$. In fact, the identity (6.1) is the result of Gauss equation. The identities (6.2), (6.3) are attained from the parallelity of $\hat{R}$, $\tilde{\sigma}$ respectively.

Fix a point $p \in N$ and put $V=T_{p}(N)$. Define a $V$-valued trilinear form \{\} on $V$ by

$$
\{X, Y, Z\}=\hat{R}(X, Y) Z+\tilde{A}_{\tilde{\sigma}(X, Y)} Z
$$

for $X, Y, Z \in V$. Then the object $(V,\{\})$ is a JTS. In fact, the condition (JT 1) is attained by (6.1) and the condition (JT 2) by (6.2), (6.3) respectively (See Ferus [3], Lemma 2 for the detailed proof). Denote also by \langle\rangle$_{L}$ the restriction of \langle\rangle$_{L}$ into $V \times V$. Then we have

$$
L(X, Y)^{t}=-\hat{R}(X, Y)+\tilde{A}_{\tilde{\sigma}(X, Y)}=\hat{R}(Y, X)+\tilde{A}_{\tilde{\sigma}(Y, X)}=L(Y, X)
$$

for $X, Y \in V$. Hence the object $\left(V,\{\},\langle\rangle_{L}\right)$ is an OJTS. Note that the tensor $\tilde{A}_{\tilde{\sigma}(*, *)}(*)$ on $N$ is parallel. Then the OJTS is independent of the choice of a point $p \in N$ by the parallelity of $\hat{R}, \tilde{A}_{\tilde{\sigma}(*, *)}(*)$.

Let $\boldsymbol{H}^{n}$ be a $2 n$-dimensional pseudo-Hermitian space with the complex structure $i_{H}$ and the pseudo-riemannian metric \langle\rangle$_{H}$, and $N^{n}$ an $n$-dimensional totally real pseudo-riemannian submanifold of $\boldsymbol{H}^{n}$. Then we have the following identities:

$$
\begin{aligned}
& \tilde{D}_{X} i_{H} Z=i_{H} \hat{V}_{X} Z \text { and thus } \tilde{R}^{\perp}(X, Y) i_{H} Z=i_{H} \hat{R}(X, Y) Z, \\
& \tilde{A}_{i_{H} X} Y=-i_{H} \tilde{\sigma}(X, Y), \\
& \left\langle\tilde{\sigma}(X, Y), i_{H} Z\right\rangle_{H}=\left\langle\tilde{\sigma}(X, Z), i_{H} Y\right\rangle_{H}
\end{aligned}
$$

for vector fields $X, Y, Z$ of $N^{n}$. In fact, the identities (6.4), (6.5) are attained by Gauss-Weingarten formulas and the identity (6.6) is attained by (6.5). 
Let $N^{n}$ be an $n$-dimensional connected totally real parallel pseudoriemannian submanifold of $\boldsymbol{H}^{n}$. Fix a point $p \in N^{n}$ and define a product on $V=T_{p}(N)$ by

$$
X \cdot Y=i_{H} \tilde{\sigma}(X, Y)
$$

for $X, Y \in V$. Denote by $A$ this algebra $(V, \cdot)$. Note that

$$
\hat{\nabla}\left(i_{H} \tilde{\sigma}\right)=0 \text { and thus } \hat{R}(X, Y) \cdot\left(i_{H} \tilde{\sigma}\right)=0
$$

for $X, Y \in V$ by the parallelity of $\tilde{\sigma}$ and (6.4).

Lemma 6.1. The object $\left(A,\langle\rangle_{H}\right)$ is an OJA. Moreover the OJTS associated with $\left(A,\langle\rangle_{H}\right)$ is the object $\left(V,\{\},\langle\rangle_{H}\right)$.

Proof. We show that $A$ is a JA. The condition (J 1 ) is obvious. Now we have

$$
\begin{aligned}
& X^{2} \cdot(X \cdot Y)-X \cdot\left(X^{2} \cdot Y\right) \\
& \quad=i_{H} \tilde{\sigma}\left(i_{H} \tilde{\sigma}(X, X), i_{H} \tilde{\sigma}(X, Y)\right)-i_{H} \tilde{\sigma}\left(X, i_{H} \tilde{\sigma}\left(i_{H} \tilde{\sigma}(X, X), Y\right)\right) \\
& \quad=-\tilde{A}_{\tilde{\sigma}(X, X)} \tilde{A}_{i_{H} X} Y+\tilde{A}_{i_{H} X} \tilde{A}_{\tilde{\sigma}(X, X)} Y=\left[\tilde{A}_{i_{H} X}, \tilde{A}_{\tilde{\sigma}(X, X)}\right](Y)
\end{aligned}
$$

for $X, Y \in V$ by (6.5), and moreover

$$
\begin{aligned}
& \left\langle\left[\tilde{A}_{i_{H} X}, \tilde{A}_{\tilde{\sigma}(X, X)}\right](Y), Z\right\rangle_{H}=\left\langle\tilde{R}^{\perp}(Y, Z) i_{H} X, \tilde{\sigma}(X, X)\right\rangle_{H} \\
& \quad=-\left\langle i_{H} \hat{R}(Y, Z) X, \tilde{\sigma}(X, X)\right\rangle_{H}=\left\langle\hat{R}(Y, Z) X, i_{H} \tilde{\sigma}(X, X)\right\rangle_{H} \\
& \quad=(1 / 3)\left\{-\left\langle X, \hat{R}(Y, Z) i_{H} \tilde{\sigma}(X, X)\right\rangle_{H}+2\left\langle X, i_{H} \tilde{\sigma}(\hat{R}(Y, Z) X, X)\right\rangle_{H}\right\} \\
& \quad=-(1 / 3)\left\langle X,\left\{\hat{R}(Y, Z) \cdot\left(i_{I I} \tilde{\sigma}\right)\right\}(X, X)\right\rangle_{H}=0
\end{aligned}
$$

for $Z \in V$ by Ricci equation and (6.6), (6.7). This implies the condition (J 2). Hence the algebra $A$ is a JA. Since

$$
\begin{aligned}
\left\langle T_{X}(Y), Z\right\rangle_{I I} & =\langle X \cdot Y, Z\rangle_{H}=\left\langle i_{H} \tilde{\sigma}(X, Y), Z\right\rangle_{H}=\left\langle i_{H} \tilde{\sigma}(X, Z), Y\right\rangle_{H} \\
& =\langle X \cdot Z, Y\rangle_{H}=\left\langle T_{X}(Z), Y\right\rangle_{H}
\end{aligned}
$$

for $X, Y, Z \in A$ by (6.6), endomorphisms $T_{X}, X \in A$, are symmetric for \langle\rangle$_{H}$. Hence the object $\left(A,\langle\rangle_{H}\right)$ is an OJA.

Let $\left(V_{A},\{\}_{A},\langle\rangle_{H}\right)$ be the OJTS associated with $\left(A,\langle\rangle_{H}\right)$. Then we have

$$
\begin{aligned}
\{X, Y, Z\}_{A} & =(X \cdot Y) \cdot Z+X \cdot(Y \cdot Z)-Y \cdot(X \cdot Z) \\
& =i_{H} \tilde{\sigma}\left(i_{H} \tilde{\sigma}(X, Y), Z\right)+i_{H} \tilde{\sigma}\left(X, i_{H} \tilde{\sigma}(Y, Z)\right)-i_{H} \tilde{\sigma}\left(Y, i_{H} \tilde{\sigma}(X, Z)\right) \\
& =\tilde{A}_{\tilde{\sigma}(X, Y)} Z+\tilde{A}_{\tilde{\sigma}(Y, Z)} X-\tilde{A}_{\tilde{\sigma}(X, Z)} Y \\
& =\tilde{A}_{\tilde{\sigma}(X, Y)} Z+\hat{R}(X, Y) Z=\{X, Y, Z\}
\end{aligned}
$$


for $X, Y, Z \in A$ by (6.1), (6.5), and thus $\left(V_{A},\{\}_{A},\langle\rangle_{H}\right)=\left(V,\{\},\langle\rangle_{H}\right)$. q.e.d.

Note that the OJA $\left(A,\langle\rangle_{H}\right)$ is independent of the choice of $p \in N^{n}$ by the parallelity of $i_{H} \tilde{\sigma}$.

Let $M^{r}$ be an $r$-dimensional connected complete totally real parallel submanifold of $\bar{M}^{r}(c), c \neq 0$. The complete inverse $\hat{M}^{r+1}$ of $M^{r}$ is a connected complete totally real parallel submanifold of $\boldsymbol{E}^{r+1}$ by Proposition 4.1, Lemma 1.1, (3), (4.5). Then we have the following

Lemma 6.2. Let $\left(A,\langle\rangle_{E}\right)$ be the OJA constructed as above from the complete inverse $\hat{M}^{r+1} \longrightarrow E^{r+1}$. Then $\left(A,\langle\rangle_{E}\right)$ has the unity $E$ and satisfies that

$\left(\mathrm{E}_{c} 1\right)$ the signature of \langle\rangle$_{E}$ is $(1, r),(0, r+1)$ according as $c<0$, $c>0$ respectively,

$\left(\mathrm{E}_{c} 2\right)\langle E, E\rangle_{E}=4 / c$.

Proof. Note that

$$
\tilde{A}_{\nu}=-(\sqrt{|c|} / 2) \mathrm{id}_{A}
$$

by (4.1), (4.6). This implies that

$$
i \tilde{\sigma}(i \nu, X)=-(\sqrt{|c|} / 2) X
$$

for $X \in A$ by (6.5). Put $E=-(2 / \sqrt{|c|}) i \nu$. Then $E$ is the unity of $A$.

The properties $\left(\mathrm{E}_{c} 1\right),\left(\mathrm{E}_{c} 2\right)$ are obvious. q.e.d

This object $\left(A,\langle\rangle_{E}\right)$ (resp. $\left(\mathfrak{g}_{A}=\sum\left(\mathfrak{g}_{A}\right)_{\mu}, \rho_{A}, J_{p_{A}},\langle\rangle_{p_{A}}\right)$ coming from $\left.\left(A,\langle\rangle_{E}\right)\right)$ is called the OJA (resp. HSGLA) associated with a complete inverse $\hat{M}^{r+1}$ and will be denoted by $\mathscr{A}_{\hat{M}}$ (resp. $\mathscr{G}_{\hat{H}}$ ).

Fix a real number $c \neq 0$. Let $\mathscr{A}=(A,\langle\rangle)$ be an OJA with unity $E$ satisfying $\left(\mathrm{E}_{c} 1\right),\left(\mathrm{E}_{c} 2\right)$ and $\mathscr{G}=\left(\mathrm{g}_{A}=\sum\left(\mathrm{g}_{A}\right)_{\mu}, \rho_{A}, J_{p_{A}},\langle\rangle_{p_{A}}\right)$ the HSGLA coming from $\mathscr{A}$. Denote by $E_{\mathscr{g}}^{r+1}$ the pseudo-Hermitian space $\left(\mathfrak{p}_{A}, J_{\mathfrak{p}_{A}}\right.$, \langle\rangle$\left._{\mathfrak{p}_{A}}\right)$. Put $N_{\mathscr{g}}^{2 r+1}(\mathrm{c} / 4)=\left\{\boldsymbol{X} \in \mathfrak{p}_{A} ;\langle\boldsymbol{X}, \boldsymbol{X}\rangle_{\mathfrak{p}_{A}}=c / 4\right\}$ and denote by $\bar{M}_{g}^{r}(c)$ the complex space form of constant holomorphic sectional curvature $c$, defined by the set of orbits in $N_{s}^{2 r+1}(c / 4)$ by the $S^{1}$-action: $\theta \rightarrow \exp \theta J_{p_{A}}$. Then $K(\nu)$ is an $(r+1)$-dimensional connected complete totally real parallel submanifold of $E_{g}^{r+1}$ and is left invariant by the $S^{1}$-action (Theorem 5.7). Since $K$ acts isometrically on $E_{s}^{r+1}$ and $\langle\nu, \nu\rangle_{p_{A}}=4 / c$, the submanifold $K(\nu)$ is contained in $N_{s}^{2 r+1}(c / 4)$. Denote by $\pi_{g}$ the projection of $N_{g}^{2 r+1}(c / 4)$ 
onto $\bar{M}_{g}^{r}(c)$. Then $M_{g}^{r}=\pi_{g}(K(\nu))$ is an $r$-dimensional connected complete totally real parallel submanifold of $\bar{M}_{\mathscr{g}}^{r}(c)$ and the complete inverse $\hat{M}_{\mathscr{g}}^{r+1}$ of $M_{\mathscr{g}}^{r}$ is the submanifold $K(\nu)$ (Lemma 1.1, (3), Proposition 4.1).

THEOREM 6.3. (1) Let $M^{r}$ be an r-dimensional connected complete totally real parallel submanifold of $\bar{M}^{r}(c)$. Then $M^{r} \hookrightarrow \bar{M}^{r}(c)$ is holomorphically congruent to $M_{\mathscr{S}_{\hat{M}}}^{r} \longrightarrow \bar{M}_{\mathfrak{S}_{\hat{M}}}^{r}(c)$, i.e., there exists a holomorphic isometry $\bar{\delta}$ of $\bar{M}^{r}(c)$ onto $\bar{M}_{\mathfrak{s}_{\hat{M}}}^{r}(c)$ such that $\bar{\delta}\left(M^{r}\right)=M_{\mathfrak{s}_{\hat{M}}}^{r}$.

(2) Let $\mathscr{A}$ be an OJA with unity $E$ satisfying $\left(\mathrm{E}_{c} 1\right),\left(\mathrm{E}_{c} 2\right)$ and $\mathscr{G}$ the HSGLA coming from $\mathscr{A}$. Then $\mathscr{A}_{\hat{H}_{g}}$ is equivalent to $\mathscr{A}$ and thus $\mathscr{G}_{\hat{\mathrm{H}}_{\mathrm{g}}}$ is equivalent to $\mathscr{G}$.

(3) Let $\mathscr{A}, \mathscr{A}^{\prime}$ be OJA's with unities $E, E^{\prime}$ satisfying $\left(\mathrm{E}_{c} 1\right),\left(\mathrm{E}_{c} 2\right)$ respectively and $\mathscr{G}, \mathscr{G}^{\prime}$ the HSGLA's coming from $\mathscr{A}, \mathscr{A}^{\prime}$ respectively. Then $\mathscr{A}$ is equivalent to $\mathscr{A}^{\prime}$ if and only if $\mathscr{G}$ is equivalent to $\mathscr{G}^{\prime}$ if and only if $M_{\mathscr{g}}^{r} \subset \bar{M}_{g}^{r}(c)$ is holomorphically congruent to $M_{\mathscr{S}^{\prime}}^{r} \longrightarrow \bar{M}_{\mathscr{S}^{\prime}}^{r}(c)$.

Proof. (1) Let $\hat{M}^{r+1}$ be the complete inverse of $M^{r}$ and fix a point $z \in \hat{M}$. Denote by $\mathscr{A}_{\hat{M}}=\left(A,\langle\rangle_{E}\right)\left(\right.$ resp. $\left.\mathscr{G}_{\hat{M}}=\left(\mathfrak{g}_{A}=\sum\left(\mathfrak{g}_{A}\right)_{\mu}, \rho_{A}, J_{\mathfrak{p}_{A}},\langle\rangle_{p_{A}}\right)\right)$ the OJA (resp. HSGLA) associated with $\hat{M}$. Identify $A \oplus i A=T_{z}(\hat{M}) \oplus$ $i T_{z}(\hat{M})$ with $E^{r+1}$.

Define a linear isomorphism $\delta$ of $E^{r+1}$ onto $\mathfrak{p}_{A}$ by

$$
\delta(X+i Y)=\left(-X,-T_{Y}, X\right) \quad \text { for } X, Y \in A .
$$

Then we have

$$
\begin{aligned}
\langle\delta(X & \left.+i Y), \delta\left(X^{\prime}+i Y^{\prime}\right)\right\rangle_{p_{A}}=\left\langle X, X^{\prime}\right\rangle_{E}+\left\langle\left(0, T_{Y}, 0\right),\left(0, T_{Y^{\prime}}, 0\right)\right\rangle_{p_{A}} \\
& =\left\langle X, X^{\prime}\right\rangle_{E}+\left\langle\left(0,(1 / 2)\left\{L_{A}(Y, E)+L_{A}(E, Y)\right\}, 0\right),\left(0, T_{Y^{\prime}}, 0\right)\right\rangle_{p_{A}} \\
& =\left\langle X, X^{\prime}\right\rangle_{E}+\left\langle T_{Y^{\prime}},(E), Y\right\rangle_{E}=\left\langle X, X^{\prime}\right\rangle_{E}+\left\langle Y, Y^{\prime}\right\rangle_{E} \\
& =\left\langle X+i Y, X^{\prime}+i Y^{\prime}\right\rangle_{E}
\end{aligned}
$$

for $X+i Y, X^{\prime}+i Y^{\prime} \in E^{r+1}$ by (5.12), and moreover

$$
\delta i(X+i Y)=\left(Y,-T_{X},-Y\right)=J_{p_{A}}\left(-X,-T_{Y}, X\right)=J_{p_{A}} \delta(X+i Y)
$$

for $X+i Y \in E^{r+1}$. Hence $\delta$ is a holomorphic isometry of the pseudoHermitian space $\boldsymbol{E}^{r+1}$ onto the pseudo-Hermitian space $\boldsymbol{E}_{\boldsymbol{g}_{\hat{M}}}^{r+1}$. This implies that $\delta\left(\hat{M}^{r+1}\right)$ is a connected complete totally real parallel submanifold of $\boldsymbol{E}_{\mathfrak{s}_{\hat{M}}}^{r+1}$ which is left invariant by the $S^{1}$-action: $\theta \rightarrow \exp \theta J_{p_{A^{*}}}$. Denote by $j$ the imbedding: $K / K_{0} \ni k K_{0} \rightarrow k(\nu) \in \mathfrak{p}_{A}$. Then the second fundamental form $\left(\tilde{\sigma}_{j}\right)_{o}$ at $o=K_{0}$ is given by 


$$
\left(\check{\sigma}_{j}\right)_{o}(A, B)=\left\{\operatorname{ad}(\boldsymbol{A}) \operatorname{ad}(\boldsymbol{B})_{\nu}\right\}_{\mathfrak{p}_{A} \cap\left(g_{A}\right)_{0}}
$$

for $A, B \in \mathfrak{m}=\{(X, 0, X) ; X \in A\}$ (See Ferus [4], Lemma 1 for the proof). Note that $\delta(z)=\nu$ by $(6.9)$ and that $T_{\nu}(K(\nu))=T_{\nu}(\delta(\hat{M}))=[\mathfrak{m}, \nu]$. Denote by $\left(\tilde{\sigma}_{K(\nu)}\right)_{\nu}$ (resp. $\left.\left(\tilde{\sigma}_{\delta(\hat{M})}\right)_{\nu}\right)$ the second fundamental form at $\boldsymbol{\nu}$ of $K(\boldsymbol{\nu}) \subset E_{\mathscr{S}_{\hat{\alpha}}}^{r+1}$ (resp. $\delta(\hat{M}) \subset \boldsymbol{E}_{\mathscr{S}_{\hat{M}}}^{r+1}$ ). Then we have

$$
\begin{aligned}
& \left(\tilde{\sigma}_{K(\nu)}\right)_{\nu}((X, 0,-X),(Y, 0,-Y))=\left(\tilde{\sigma}_{j}\right)_{o}((X, 0, X),(Y, 0, Y)) \\
& \quad=\left(0,(1 / 2)\left\{L_{A}(X, Y)+L_{A}(Y, X)\right\}, 0\right)=\left(0, T_{X \cdot Y}, 0\right)=-\delta(i X \cdot Y) \\
& \quad=\delta\left(\tilde{\sigma}_{z}(X, Y)\right)=\left(\tilde{\sigma}_{\delta(M)}\right)_{\nu}((X, 0,-X),(Y, 0,-Y))
\end{aligned}
$$

for $(X, 0,-X),(Y, 0,-Y) \in T_{\nu}(K(\nu))=T_{\nu}(\delta(\hat{M}))$ and thus

$$
\left(\tilde{\sigma}_{K(\nu)}\right)_{\nu}=\left(\tilde{\sigma}_{\delta(\tilde{H})}\right)_{\nu} .
$$

Let $\bar{\delta}$ be a holomorphic isometry of $\bar{M}^{r}(c)$ onto $\bar{M}_{\mathfrak{s}_{\hat{M}}}^{r}(c)$, induced by $\delta$. Then we note that $\bar{\delta}\left(M^{r}\right)=\pi_{\mathscr{g}_{\hat{H}}}\left(\delta\left(\hat{M}^{r+1}\right)\right)$. Put $p=\pi_{\mathscr{s}_{\hat{M}}}(\nu)$ and denote by $\left(\sigma_{M_{\mathscr{g}_{\hat{M}}}}\right)_{p}$ (resp. $\left.\left(\sigma_{\tilde{\delta}(M)}\right)_{p}\right)$ the second fundamental form at $p$ of $M_{g_{M}} \hookrightarrow \bar{M}_{\mathfrak{s}_{\hat{g}}}^{r}(c)$ (resp. $\left.\bar{\delta}(M) \smile \bar{M}_{\mathscr{S}_{\mathcal{M}}}^{r}(c)\right)$. Then we have

$$
\left(\sigma_{M_{\mathcal{S}_{\mathcal{M}}}}\right)_{p}=\left(\sigma_{\tilde{\delta}(M)}\right)_{p}
$$

by (6.10), (4.1), (4.6), Lemma 1.1, (1). Hence we have $\bar{\delta}(M)=M_{\mathfrak{s}_{\hat{H}}}^{r}$ (Naitoh [8], Lemma 3.2).

(2) Let $\mathscr{A}=(A,\langle\rangle)$ and $\mathscr{G}=\left(\mathfrak{g}_{A}=\sum\left(\mathfrak{g}_{A}\right)_{\mu}, \rho_{A}, J_{p_{A}},\langle\rangle_{p_{A}}\right)$. Then the second fundamental form $\left(\tilde{\sigma}_{\hat{M}_{g}}\right)_{\nu}$ at $\nu$ of $\hat{M}_{\mathscr{g}}=K(\boldsymbol{\nu}) \subset \boldsymbol{E}_{g}^{r+1}$ is given by

$$
\left(\tilde{\sigma}_{\hat{M}_{g}}\right)_{\nu}(A, B)=\left(0, T_{X \cdot Y}, 0\right)
$$

for $A=(X, 0,-X), B=(Y, 0,-Y)$ and thus

$$
J_{p_{A}}\left(\tilde{\sigma}_{\hat{M}_{g}}\right)_{\nu}(A, B)=(-X \cdot Y, 0, X \cdot Y) .
$$

Define a linear isomorphism $g$ of $A$ onto [m, ע] by $g(X)=(-X, 0, X)$ for $X \in A$. Then we have

$$
\begin{aligned}
g(X \cdot Y) & =(-X \cdot Y, 0, X \cdot Y)=J_{p_{A}}\left(\tilde{\sigma}_{\hat{M}_{g}}\right)_{\nu}(A, B) \\
& =J_{p_{A}}\left(\tilde{\sigma}_{\hat{M}_{g}}\right)_{\nu}(g(X), g(Y))
\end{aligned}
$$

and moreover

$$
\langle g(X), g(Y)\rangle_{p_{A}}=\langle X, Y\rangle \text {. }
$$

This implies that $g$ is an isomorphism of OJA $\mathscr{A}$ onto OJA $\mathscr{A}_{\hat{M}_{\mathcal{G}}}$ and thus 
$\mathscr{A}$ is equivalent to $\mathscr{A}_{\hat{M}_{\mathscr{G}}}$. Hence $\mathscr{G}$ is equivalent to $\mathscr{G}_{\hat{H}_{\mathscr{G}}}$ by Theorem 5.5, (2).

(3) It is obvious by Theorem $5.5,(2)$ that $\mathscr{A}$ is equivalent to $\mathscr{A}^{\prime}$ if and only if $\mathscr{G}$ is equivalent to $\mathscr{G}^{\prime}$.

Assume that $\mathscr{A}=(A,\langle\rangle)$ is equivalent to $\mathscr{A}^{\prime}=\left(A^{\prime},\langle\rangle^{\prime}\right)$, i.e., there exists an algebra isomorphism $\alpha$ of $A$ onto $A^{\prime}$ such that $\langle\alpha(X), \alpha(Y)\rangle^{\prime}$ $=\langle X, Y\rangle$ for $X, Y \in A$. The isomorphism $\alpha$ induces the isomorphism $\tau_{g_{\alpha}}$ of $\mathscr{G}$ onto $\mathscr{G}^{\prime}$. Then we have

$$
\tau_{g_{\alpha}}(\nu)=\tau_{g_{\alpha}}\left(0,-\mathrm{id}_{A}, 0\right)=\left(0,-g_{\alpha} \circ \mathrm{id}_{A^{\prime}} \circ g_{\alpha}^{-1}, 0\right)=\left(0,-\mathrm{id}_{A^{\prime}}, 0\right)=\nu^{\prime} .
$$

The restriction $\delta$ of $\tau_{g_{\alpha}}$ into $\mathfrak{p}_{A}$ is a holomorphic isometry of $\boldsymbol{E}_{\mathscr{g}}^{r+1}$ onto $\boldsymbol{E}_{\boldsymbol{g}^{\prime}}^{r+1}$ and $\delta(K(\nu))=K^{\prime}\left(\boldsymbol{\nu}^{\prime}\right)$. Hence we have $\bar{\delta}\left(M_{\mathscr{g}}^{r}\right)=M_{\mathscr{g}^{\prime}}^{r}$, where $\bar{\delta}$ denotes a holomorphic isometry of $\bar{M}_{g}^{r}(c)$ onto $\bar{M}_{g}^{r}(c)$, induced by $\delta$.

Conversely, assume that $M_{\mathfrak{G}}^{r} \longrightarrow \bar{M}_{g}^{r}(c)$ is holomorphically congruent to $M_{\mathscr{g}^{\prime}}^{r} \subset \bar{M}_{g^{\prime}}^{r}(c)$, i.e., there exists a holomorphic isometry $\bar{\delta}$ of $\bar{M}_{\mathscr{g}}^{r}(c)$ onto $\bar{M}_{g^{\prime}}^{r}(c)$ such that $\bar{\delta}\left(M_{g}^{r}\right)=M_{g^{\prime}}^{r}$. Then $\bar{\delta}$ induces a holomorphic isometry $\delta$ of $\boldsymbol{E}_{g}^{r+1}$ onto $\boldsymbol{E}_{g^{\prime}}^{r+1}$ such that $\delta\left(\hat{M}_{g}^{r+1}\right)=\hat{M}_{g^{\prime}}^{r+1}$. Denote by $\tilde{\sigma}_{\mathscr{g}}$ (resp. $\left.\tilde{\sigma}_{g^{\prime}}\right)$ the second fundamental form of $\hat{M}_{\mathscr{g}}^{r+1} \subset E_{\mathscr{g}}^{r+1}$ (resp. $\hat{M}_{\mathscr{F}^{\prime}}^{r+1} \longrightarrow \boldsymbol{E}_{\mathscr{g}^{\prime}}^{r+1}$ ). Then we have

$$
\delta\left(J_{p_{A}} \tilde{\sigma}_{s}(X, Y)\right)=J_{p_{A}} \delta\left(\tilde{\sigma}_{s}(X, Y)\right)=J_{p_{A}} \tilde{\sigma}_{\mathscr{F}^{\prime}}(\delta X, \delta Y)
$$

for $X, Y \in T_{z}\left(\hat{M}_{g}^{r+1}\right)$. Hence $\mathscr{A}_{\hat{M}_{g}}$ is equivalent to $\mathscr{A}_{\hat{M}_{g^{\prime}} \cdot}$ This implies that $\mathscr{A}$ is equivalent to $\mathscr{A}^{\prime}$ by (2).

q.e.d.

Remark 6.4. Theorem 6.3 implies that the classification of $r$-dimensional connected complete totally real parallel submanifold of $\bar{M}^{r}(c), c \neq 0$, reduces to that of HSGLA's associated with OJA's with unities satisfying $\left(\mathrm{E}_{c} 1\right),\left(\mathrm{E}_{c} 2\right)$.

Remark 6.5. The proof of Theorem 6.3, (2) doesn't need the conditions $\left(\mathrm{E}_{c} 1\right),\left(\mathrm{E}_{c} 2\right)$ for $\mathscr{A}$. Hence the claim (2) is true for any OJA with unity.

Let $\mathscr{A}=(A,\langle\rangle)$ be an OJA with unity and $\mathscr{G}=\left(\mathfrak{g}_{A}=\sum\left(\mathfrak{g}_{A}\right)_{\mu}, \rho_{A}, J_{p_{A}}\right.$, \langle\rangle$\left._{p_{A}}\right)$ the HSGLA coming from $\mathscr{A}$. Denote by $\boldsymbol{H}_{g}^{r+1}$ the pseudo-Hermitian space $\left(\mathfrak{p}_{A}, J_{p_{A}},\langle\rangle_{p_{A}}\right)$ and put $\hat{M}_{g}^{r+1}=K(\nu)$. Then the proof of Theorem 6.3 , (3) implies that the claim (3) can be generalized as follows: Let $\mathscr{A}$, $\mathscr{A}^{\prime}$ be OJA's with unity and $\mathscr{G}, \mathscr{G}^{\prime}$ the HSGLA's coming from $\mathscr{A}, \mathscr{A}^{\prime}$ respectively. Then $\mathscr{A}$ is equivalent to $\mathscr{A}^{\prime}$ if and only if $\mathscr{G}$ is equivalent to $\mathscr{G}^{\prime}$ if and only if $\hat{M}_{\mathscr{g}}^{r+1} \smile \boldsymbol{H}_{\mathcal{G}}^{r+1}$ is holomorphically and linearly con- 
gruent to $\hat{M}_{\mathscr{g}^{\prime}}^{r+1} \longrightarrow \boldsymbol{H}_{\mathscr{g}^{\prime}}^{r+1}$, i.e., there exists a holomorphic and linear isometry $\delta$ of $\boldsymbol{H}_{\mathscr{g}}^{r+1}$ onto $\boldsymbol{H}_{\mathscr{g}^{\prime}}^{r+1}$ such that $\delta\left(\hat{M}_{\mathscr{g}}^{r+1}\right)=\hat{M}_{\mathscr{g}^{\prime}}^{r+1}$.

\section{§7. A decomposition of the HSGLA coming from an OJA with unity}

Firstly we define the following two notions for each category given in this series: one is "the sum of objects" and the other is "a decomposition of an object".

Let $\left(V_{i},\{\}_{i}\right), 1 \leqq i \leqq s$, be JTS's. Put $V=\oplus_{i=1}^{s} V_{i}$ and define a $V$-valued trilinear form \{\} on $V$ by

$$
\left\{\sum X_{i}, \sum Y_{i}, \sum Z_{i}\right\}=\sum\left\{X_{i}, Y_{i}, Z_{i}\right\}_{i}
$$

for $X_{i}, Y_{i}, Z_{i} \in V_{i}, 1 \leqq i \leqq s$. Then the object $(V,\{\})$ is a JTS. This JTS is called the sum of JTS's $\left(V_{i},\{\}_{i}\right)$ and is denoted by $(V,\{\})=$ $\oplus\left(V_{i},\{\}_{i}\right)$. Conversely, let $(V,\{\})$ be a JTS. Let $V=\oplus_{i=1}^{s} V_{i}$ be the direct sum of linear subspaces $V_{i}$ satisfying that $\left\{V_{i}, V_{j}, V_{k}\right\} \subset V_{i} \cap V_{j}$ $\cap V_{k}$ for $1 \leqq i, j, k \leqq s$. Denote by \{\}$_{i}, 1 \leqq i \leqq s$, the restrictions of \{\} into subspaces $V_{i}$ respectively. Then the objects $\left(V_{i},\{\}_{i}\right), 1 \leqq i \leqq s$, are JTS's and the JTS $(V,\{\})$ is equivalent to $\oplus\left(V_{i},\{\}_{i}\right)$. The sum $\oplus\left(V_{i},\{\}_{i}\right)$ is called a decomposition of the $\operatorname{JTS}(V,\{\})$.

Let $\mathscr{V}_{i}=\left(V_{i},\{\}_{i},\langle\rangle_{i}\right), 1 \leqq i \leqq s$, be OJTS's. Let $(V,\{\})$ be the sum of JTS's $\left(V_{i},\{\}_{i}\right)$ and define a non-degenerate symmetric bilinear form $\langle>$ on $V$ by

$$
\left\langle\sum X_{i}, \sum Y_{i}\right\rangle=\sum\left\langle X_{i}, Y_{i}\right\rangle_{i}
$$

for $X_{i}, Y_{i} \in V_{i}, 1 \leqq i \leqq s$. Then the object $\mathscr{V}=(V,\{\},\langle>)$ is an OJTS. This OJTS is called the sum of OJTS's $\mathscr{V}_{i}$ and is denoted by $\mathscr{V}=\oplus \mathscr{V}_{i}$. Conversely, let $\mathscr{V}=(V,\{\},\langle\rangle)$ be an OJTS. Let $\oplus\left(V_{i},\{\}_{i}\right)$ be a decomposition of the JTS $(V,\{\})$ such that $\left\langle V_{i}, V_{j}\right\rangle=\{0\}$ for $i \neq j$. Denote by \langle\rangle$_{i}, 1 \leqq i \leqq s$, the restrictions of $\left\langle>\right.$ into subspaces $V_{i}$ respectively. Then the objects $\mathscr{V}_{i}=\left(V_{i},\{\}_{i},\langle\rangle_{i}\right), 1 \leqq i \leqq s$, are OJTS's and $\mathscr{V}$ is equivalent to $\oplus \mathscr{V}_{i}$. The sum $\oplus \mathscr{V}_{i}$ is called a decomposition of the OJTS $\mathscr{V}$.

Let $\mathscr{A}_{i}=\left(A_{i},\langle\rangle_{i}\right), 1 \leqq i \leqq s$, be OJA's. Let $A=\oplus A_{i}$ be the sum of JA's $A_{i}$ and define a non-degenerate symmetric bilinear form $\langle>$ on $A$ by

$$
\left\langle\sum X_{i}, \sum Y_{i}\right\rangle=\sum\left\langle X_{i}, Y_{i}\right\rangle_{i}
$$


for $X_{i}, Y_{i} \in A_{i}, 1 \leqq i \leqq s$. Then the object $\mathscr{A}=(A,\langle\rangle)$ is an OJA. This OJA is called the sum of OJA's $\left(A_{i},\langle\rangle_{i}\right)$ and is denoted by $\mathscr{A}=$ $\oplus \mathscr{A}_{i}$. Conversely, let $\mathscr{A}=(A,\langle\rangle)$ be an OJA. Let $\oplus A_{i}$ be a decomposition of $A$ into the sum of ideals $A_{i}$ satisfying that $\left\langle A_{i}, A_{j}\right\rangle=\{0\}$ for $i \neq j$. Denote by \langle\rangle$_{i}, 1 \leqq i \leqq s$, the restrictions of $\left\langle>\right.$ into ideals $A_{i}$ respectively. Then the objects $\mathscr{A}_{i}=\left(A_{i},\langle\rangle_{i}\right), 1 \leqq i \leqq s$, are OJA's and $\mathscr{A}$ is equivalent to $\oplus \mathscr{A}_{i}$. The sum $\oplus \mathscr{A}_{i}$ is called a decomposition of the OJA $\mathscr{A}$.

Moreover, two notions of "sum" and "decomposition" can be defined naturally for other categories: OSLA, HSLA, OSGLA, HSGLA, etc. Since the definitions are clear, they are not discribed here. But the notions are often used in this paper.

Proposition 7.1. (1) Let $\mathscr{V}, \mathscr{V}_{1}, \mathscr{V}_{2}$ be OJTS's and $\mathscr{G}, \mathscr{G}_{1}, \mathscr{G}_{2}$ the OSGLA's associated with $\mathscr{V}, \mathscr{V}_{1}, \mathscr{V}_{2}$ respectively. Then $\mathscr{V}$ is equivalent to $\mathscr{V}_{1} \oplus \mathscr{V}_{2}$ if and only if $\mathscr{G}$ is equivalent to $\mathscr{G}_{1} \oplus \mathscr{G}_{2}$.

(2) (a) Let $\mathscr{A}_{1}, \mathscr{A}_{2}$ be OJA's. Then $\mathscr{A}_{1} \oplus \mathscr{A}_{2}$ has the unity if and only if each $\mathscr{A}_{i}$ has the unity.

(b) Let $\mathscr{G}_{1}, \mathscr{G}_{2}$ be HSGLA's. Then $\mathscr{G}_{1} \oplus \mathscr{G}_{2}$ is equivalent to an HSGLA coming from an OJA with unity if and only if each $\mathscr{G}_{i}$ is equivalent to an HSGLA coming from an OJA with unity.

(3) Let $\mathscr{A}, \mathscr{A}_{1}, \mathscr{A}_{2}$ be OJA's with unity and $\mathscr{G}, \mathscr{G}_{1}, \mathscr{G}_{2}$ the HSGLA's coming from $\mathscr{A}, \mathscr{A}_{1}, \mathscr{A}_{2}$ respectively. Then $\mathscr{A}$ is equivalent to $\mathscr{A}_{1} \oplus \mathscr{A}_{2}$ if and only if $\mathscr{G}$ is equivalent to $\mathscr{G}_{1} \oplus \mathscr{G}_{2}$.

Proof. (1) Note that the OSGLA associated with $\mathscr{V}_{1} \oplus \mathscr{V}_{2}$ is equivalent to $\mathscr{G}_{1} \oplus \mathscr{G}_{2}$. Then our claim is clear by Theorem 5.4, (2).

(2) The claim (a) is obvious. We show the claim (b). Assume that $\mathscr{G}_{1} \oplus \mathscr{G}_{2}$ is equivalent to $\mathscr{G}=\left(\mathfrak{g}_{A}=\sum\left(\mathfrak{g}_{A}\right)_{\mu}, \rho_{A}, J_{p_{A}},\langle\rangle_{p_{A}}\right)$ coming from $\mathscr{A}=(A,\langle\rangle)$ with the unity $E$ by an isomorphism $\tau: \mathscr{G}_{1} \oplus \mathscr{G}_{2} \Im \mathscr{G}$. Let $\mathscr{G}_{i}=\left(\mathfrak{g}_{i}=\sum\left(\mathfrak{g}_{i}\right)_{\mu}, \rho_{i}, J_{p_{i}},\langle\rangle_{p_{i}}\right), i=1,2$. Put $A_{i}=\left\{X \in A ;(X, 0,0) \in \tau\left(\mathfrak{g}_{i}\right)\right\}$, $i=1,2$. Then $A$ is decomposed into the sum of linear subspaces $A_{i}$. We show that $A_{i}, i=1,2$, are orthogonal ideals of the JA $A$ for $\langle>$. Suppose that $X \in A_{i}$, i.e., $(X, 0,0) \in \tau\left(\mathfrak{g}_{i}\right)$. Since $\tau\left(\mathfrak{g}_{i}\right)$ is an ideal of $\mathfrak{g}_{A}$, we have

$$
(X \cdot Y, 0,0)=\left(L_{A}(X, Y) E, 0,0\right)=-2[[(X, 0,0),(0,0, Y)],(E, 0,0)] \in \tau\left(\mathfrak{g}_{i}\right)
$$

for $Y \in A$ and thus $X \cdot Y \in A_{i}$. This implies that $A_{i}$ is an ideal of $A$. 
Moreover, noting that $\left(X_{i}, 0,-X_{i}\right) \in \tau\left(\mathfrak{p}_{i}\right)$ for $X_{i} \in A_{i}, i=1,2$, we have

$$
\left\langle X_{1}, X_{2}\right\rangle=\left\langle\left(X_{1}, 0,-X_{1}\right),\left(X_{2}, 0,-X_{2}\right)\right\rangle_{\mathfrak{p}}=0
$$

and thus $\left\langle A_{1}, A_{2}\right\rangle=\{0\}$. Denote by \langle\rangle$_{i}, i=1,2$, the restrictions of \langle\rangle into the ideals $A_{i}$ respectively and put $\mathscr{A}_{i}=\left(A_{i},\langle\rangle_{i}\right)$ for $i=1,2$. Then OJA's $\mathscr{A}_{i}$ have the unities by (a) and the OJA $\mathscr{A}$ is decomposed into the $\operatorname{sum} \mathscr{A}_{1} \oplus \mathscr{A}_{2}$.

Let $\mathscr{G}_{i}^{\prime}=\left(\mathfrak{g}_{i}^{\prime}=\sum\left(\mathfrak{g}_{i}^{\prime}\right)_{\mu}, \rho_{i}^{\prime}, J_{\mathfrak{p}_{i}^{\prime}},\langle\rangle_{\mathfrak{p}_{i}^{\prime}}\right), i=1,2$, be the HSGLA's coming from $\mathscr{A}_{i}$ respectively. Denote by $F_{i}, i=1,2$, the restrictions of $F \in L_{\Lambda}$ into $A_{i}$ respectively. Then we have

$$
\left(\mathfrak{g}_{i}^{\prime}\right)_{0}=\left\{\left(0,\left(L_{A}(X, Y)\right)_{i}, 0\right) ; X, Y \in A_{i}\right\}
$$

Define mappings $\lambda_{i}, i=1,2$, of $g_{i}^{\prime}$ into $\tau\left(g_{i}\right)$ by

$$
\lambda_{i}\left(\left(X_{i},\left(L_{A}\left(Z_{i}, W_{i}\right)\right)_{i}, Y_{i}\right)\right)=\left(X_{i}, L_{A}\left(Z_{i}, W_{i}\right), Y_{i}\right)
$$

for $X_{i}, Y_{i}, Z_{i}, W_{i} \in A_{i}, i=1,2$. Then we can easily see that $\lambda_{i}$ are welldefined isomorphisms of the HSGLA's $\mathscr{G}_{i}^{\prime}$ onto the HSGLA's $\tau\left(\mathscr{G}_{i}\right)$ respectively. Hence $\mathscr{G}_{i}$ are equivalent to the HSGLA's $\mathscr{G}_{i}^{\prime}$ coming from $\mathscr{A}_{i}$ respectively.

The converse is obvious.

(3) Note that the HSGLA coming from $\mathscr{A}_{1} \oplus \mathscr{A}_{2}$ is equivalent to $\mathscr{G}_{1} \oplus \mathscr{G}_{2}$. Then our claim is clear by Theorem $5.5,(2)$. q.e.d.

Let $\mathscr{V}=(V,\{\},\langle\rangle)$ be an OJTS and denote by $\beta$ the trace form of the JTS $(V,\{\})$. Then $\beta$ is a symmetric bilinear form on $V$. Define a symmetric endomorphism $L_{r}$ of $V$ by

$$
\beta(X, Y)=\left\langle L_{r}(X), Y\right\rangle
$$

for $X, Y \in V$. Let $\left\{e_{1}, \cdots, e_{k}, e_{k+1}, \cdots, e_{n}\right\}$ be an orthonormal basis of $V$, i.e., $\left\langle e_{i}, e_{i}\right\rangle=-1$ for $1 \leqq i \leqq k,\left\langle e_{j}, e_{j}\right\rangle=1$ for $k+1 \leqq j \leqq n$, and $\left\langle e_{i}, e_{j}\right\rangle$ $=0$ for $1 \leqq i \neq j \leqq n$. Then the symmetric endomorphism $L_{r}$ is given by

$$
L_{\curlyvee}=-\sum_{i=1}^{k} L\left(e_{i}, e_{i}\right)+\sum_{j=k+1}^{n} L\left(e_{j}, e_{j}\right) .
$$

In fact, note that $\langle L(X, Y) Z, W\rangle=\langle L(Z, W) X, Y\rangle$ for $X, Y, Z, W \in V$ by (5.3), (JT 1). Then we have

$$
\begin{aligned}
\beta(X, Y) & =\operatorname{Tr} L(X, Y)=-\sum_{i=1}^{k}\left\langle L(X, Y) e_{i}, e_{i}\right\rangle+\sum_{j=k+1}^{n}\left\langle L(X, Y) e_{j}, e_{j}\right\rangle \\
& =-\sum_{i=1}^{k}\left\langle L\left(e_{i}, e_{i}\right) X, Y\right\rangle+\sum_{j=k+1}^{n}\left\langle L\left(e_{j}, e_{j}\right) X, Y\right\rangle \\
& =\left\langle\left(-\sum_{i=1}^{k} L\left(e_{i}, e_{i}\right)+\sum_{j=k+1}^{n} L\left(e_{j}, e_{j}\right)\right) X, Y\right\rangle
\end{aligned}
$$


for $X, Y \in V$. This implies (7.1).

Moreover the symmetric endomorphism $L_{r}$ is contained in the center of $L$, i.e.,

$$
\left[L_{r}, L(X, Y)\right]=0
$$

for $X, Y \in V$. In fact, note that $\beta(L(X, Y) Z, W)=\beta(Z, L(Y, X) W)$ for $X, Y$, $Z, W \in V$ by (JT 2). Then we have

$$
\begin{aligned}
\left\langle\left[L_{r}, L(X, Y)\right](Z), W\right\rangle & =\left\langle L(X, Y) Z, L_{r}(W)\right\rangle-\left\langle L_{r}(Z), L(Y, X) W\right\rangle \\
& =\beta(L(X, Y) Z, W)-\beta(Z, L(Y, X) W)=0
\end{aligned}
$$

for $X, Y, Z, W \in V$. This implies (7.2).

Let $\mathscr{V}=(V,\{\},\langle\rangle)$ be an OJTS and $\left(V_{1},\{\}_{1}\right) \oplus\left(V_{2},\{\}_{2}\right)$ a decomposition of the JTS $(V,\{\})$. Denote by \langle\rangle$_{i}, i=1,2$, the restrictions of $\left\langle>\right.$ into subspaces $V_{i}$ respectively.

LEMma 7.2. Assume that the symmetric endomorphism $L_{r}$ is nondegenerate on $V_{1}$. Then the objects $\mathscr{V}_{i}=\left(V_{i},\{\}_{i},\langle\rangle_{i}\right), i=1,2$, are OJTS's and the OJTS $\mathscr{V}$ is equivalent to the sum $\mathscr{V}_{1} \oplus \mathscr{V}_{2}$.

Proof. It is sufficient to show that $\left\langle V_{1}, V_{2}\right\rangle=\{0\}$. Note that $\beta\left(V_{1}, V_{2}\right)$ $=\{0\}$ and thus $\left\langle L_{\mathscr{r}}\left(V_{1}\right), V_{2}\right\rangle=\{0\}$. Since $L_{\mathfrak{r}}$ is non-degenerate on $V_{1}$, we have $L_{\Upsilon}\left(V_{1}\right)=V_{1}$ and thus $\left\langle V_{1}, V_{2}\right\rangle=\{0\}$.

q.e.d.

Let $\mathscr{V}=(V,\{\},\langle\rangle)$ be an OJTS. Put $V_{i}=\left(L_{r}\right)^{i}(V)$ for non-negative integers $i$. Then we have a decreasing sequence:

$$
V=V_{0} \supseteqq V_{1} \supseteqq \cdots \supseteqq V_{i} \supseteqq \cdots
$$

Let $\ell$ be the least number of $i$ 's such that $V_{i}=V_{i+1}$, and put $V_{\text {non }}=V_{\ell}, V_{\text {deg }}$ $=\operatorname{Ker}\left(L_{r}\right)^{\ell}$. Note that $L_{r}\left(V_{\text {non }}\right)=V_{\text {non }}$. Then we have the direct sum $V=V_{\text {deg }} \oplus V_{\text {non }}$. Denote by \{\}$_{\text {deg }},\{\}_{\text {non }}$ (resp. \langle\rangle$_{\text {deg }},\langle\rangle_{\text {non }}$ ) the restrictions of \{\} (resp. \langle\rangle ) into $V_{\text {deg }}, V_{\text {non }}$ respectively.

Proposition 7.3. (1) The objects $\mathscr{V}_{\text {deg }}=\left(V_{\text {deg }},\{\}_{\text {deg }},\langle\rangle_{\text {deg }}\right), \mathscr{V}_{\text {non }}=$ ( $V_{\text {non }},\{\}_{\text {non }},\langle\rangle_{\text {non }}$ ) are OJTS's and the OJTS $\mathscr{V}$ is equivalent to the sum $\mathscr{V}_{\text {deg }} \oplus \mathscr{V}_{\text {non }}$.

(2) The symmetric endomorphisms $L_{\gamma_{\mathrm{deg}}}, L_{\mathscr{r}_{\mathrm{non}}}$ are the restrictions of $L_{r}$ into subspaces $V_{\text {deg }}, V_{\text {non }}$ respectively. Hence,

(a) $\left(L_{r_{\mathrm{deg}}}\right)^{\ell}=0$,

(b) $L_{\mathscr{r}_{\text {non }}}$ is non-degenerate, and thus the JTS underlying $\mathscr{V}_{\text {non }}$ is nondegenerate. 
(3) Let $\mathscr{G}, \mathscr{G}_{\mathrm{deg}}, \mathscr{G}_{\text {non }}$ be the OSGLA's associated with $\mathscr{V}, \mathscr{V}_{\mathrm{deg}}, \mathscr{V}_{\text {non }}$ respectively. Then $\mathscr{G}$ is equivalent to the sum $\mathscr{G}_{\mathrm{deg}} \oplus \mathscr{G}_{\mathrm{non}}$. Moreover the Lie algebra $\mathfrak{g}_{\mathrm{deg}}$ underlying $\mathscr{G}_{\mathrm{deg}}$ is not semi-simple and the Lie algebra $\mathfrak{g}_{\mathrm{non}}$ underlying $\mathscr{G}_{\text {non }}$ is semi-simple.

(4) Let $\mathscr{V}, \mathscr{V}^{\prime}$ be OJTS's and $\mathscr{G}, \mathscr{G}^{\prime}$ the OSGLA's associated with $\mathscr{V}$, $\mathscr{V}^{\prime}$ respectively. Then the following four statements are equivalent to one another:

(a) $\mathscr{V}$ is equivalent to $\mathscr{V}^{\prime}$.

(b) $\mathscr{V}_{\mathrm{deg}}, \mathscr{V}_{\text {non }}$ are equivalent to $\mathscr{V}_{\mathrm{deg}}^{\prime}, \mathscr{V}_{\text {non }}^{\prime}$ respectively.

(c) $\mathscr{G}$ is equivalent to $\mathscr{G}^{\prime}$.

(d) $\mathscr{G}_{\mathrm{deg}}, \mathscr{G}_{\text {non }}$ are equivalent to $\mathscr{G}_{\mathrm{deg}}^{\prime}, \mathscr{G}_{\mathrm{non}}^{\prime}$ respectively.

Proof. (1) Note that

$$
L(V, V) V_{\mathrm{deg}} \subset V_{\mathrm{deg}}, L(V, V) V_{\mathrm{non}} \subset V_{\mathrm{non}}
$$

by (7.2), and moreover

$$
L\left(V_{\mathrm{deg}}, V\right) V_{\text {non }}=L\left(V_{\text {non }}, V\right) V_{\text {deg }}=\{0\}
$$

by (7.3), (JT 1). Since $L\left(L_{r}(X), Y\right)=L\left(X, L_{r}(Y)\right)$ for $X, Y \in V$ by (7.2), (JT 2), we have

$$
L\left(V_{\text {deg }}, V_{\text {non }}\right)=L\left(V_{\text {non }}, V_{\text {deg }}\right)=\{0\} .
$$

Hence, by $(7.3) \sim(7.5)$, the objects $\left(V_{\text {deg }},\{\}_{\text {deg }}\right),\left(V_{\text {non }},\{\}_{\text {non }}\right)$ are JTS's and the JTS $(V,\{\})$ is decomposed into the sum $\left(V_{\text {deg }},\{\}_{\text {deg }}\right) \oplus\left(V_{\text {non }},\{\}_{\text {non }}\right)$. Note that $L_{\mathscr{r}}$ is non-degenerate on $V_{\text {non. }}$. Then, by Lemma 7.2, the objects $\mathscr{V}_{\text {deg }}, \mathscr{V}_{\text {non }}$ are OJTS's and the OJTS $\mathscr{V}$ is equivalent to the sum $\mathscr{V}_{\text {deg }} \oplus \mathscr{V}_{\text {non }}$.

The claim (2) is clear by (7.1), (7.4) and the claim (3) by Proposition $7.1,(1)$ and the claims (1), (2).

(4) We show that (a) $\Rightarrow\left(\right.$ b). Assume that $\mathscr{V}$ is equivalent to $\mathscr{V}^{\prime}$ by an isomorphism $g: \mathscr{V} \widetilde{\mathscr{V}^{\prime}}$. Then we have $L_{\gamma^{\prime}}=g \circ L_{r^{\prime}} \circ g^{-1}$ by (7.1). Hence the restriction of $g$ into $V_{\text {deg }}\left(\right.$ resp. $V_{\text {non }}$ ) is an isomorphism of $\mathscr{V}_{\text {deg }}$ (resp. $\mathscr{V}_{\text {non }}$ ) onto $\mathscr{V}_{\text {deg }}^{\prime}$ (resp. $\mathscr{V}_{\text {non }}^{\prime}$ ).

The claims that $(b) \Rightarrow(d),(c) \Rightarrow$ (a) are obvious by Theorem $5.4,(2)$ and the claim that $(d) \Rightarrow$ (c) by the claim (3).

Let $\mathscr{A}=(A,\langle\rangle)$ be an OJA with the unity $E$ and $\mathscr{G}=\left(\mathfrak{g}_{A}=\sum\left(\mathfrak{g}_{A}\right)_{\mu}\right.$, $\left.\rho_{A}, J_{p_{A}},\langle\rangle_{\mathfrak{p}_{A}}\right)$ the HSGLA coming from $\mathscr{A}$. Denote by $\mathscr{V}(\mathscr{A})$ the OJTS $\left(V_{A},\{\}_{A},\langle\rangle\right)$ associated with $\mathscr{A}$ and by $\mathcal{O}(\mathscr{G})$ the OSGLA $\left(\mathfrak{g}_{A}=\sum\left(\mathfrak{g}_{A}\right)_{\mu}\right.$, $\rho_{A},\langle\rangle_{p_{A}}$ ) underlying $\mathscr{G}$. Then the OSGLA $\mathcal{O}(\mathscr{G})$ is associated with the 
OJTS $\mathscr{V}(\mathscr{A})$. Regard $\left(V_{A}\right)_{\mathrm{deg}},\left(V_{A}\right)_{\text {non }}$ as subspaces of $A$ and denote them by $A_{\text {deg }}, A_{\text {non }}$ respectively.

Proposition 7.4. (1) The objects $\mathscr{A}_{\mathrm{deg}}=\left(A_{\mathrm{deg}},\langle\rangle_{\mathrm{deg}}\right), \mathscr{A}_{\mathrm{non}}=\left(A_{\mathrm{non}}\right.$, \langle\rangle$_{\text {non }}$ ) are OJA's with unity and the OJA $\mathscr{A}$ is decomposed into the sum $\mathscr{A}_{\mathrm{deg}} \oplus \mathscr{A}_{\mathrm{non}}$. Hence the HSGLA $\mathscr{G}$ is equivalent to the sum $\mathscr{G}_{\mathrm{deg}} \oplus \mathscr{G}_{\mathrm{non}}$ of the HSGLA's $\mathscr{G}_{\mathrm{deg}}, \mathscr{G}_{\mathrm{non}}$ coming from $\mathscr{A}_{\mathrm{deg}}, \mathscr{A}_{\mathrm{non}}$ respectively.

(2) The OJTS's $\mathscr{V}\left(\mathscr{A}_{\mathrm{deg}}\right), \mathscr{V}\left(\mathscr{A}_{\mathrm{non}}\right)$ are equivalent to the OJTS's $\mathscr{V}(\mathscr{A})_{\mathrm{deg}}$, $\mathscr{V}(\mathscr{A})_{\text {non }}$ respectively. Hence the OSGLA's $\mathcal{O}\left(\mathscr{G}_{\mathrm{deg}}\right), \mathcal{O}\left(\mathscr{G}_{\mathrm{non}}\right)$ are equivalent to the OSGLA's $\mathcal{O}(\mathscr{G})_{\mathrm{deg}}, \mathcal{O}(\mathscr{G})_{\mathrm{non}}$ respectively.

(3) Let $\mathscr{A}, \mathscr{A}^{\prime}$ be OJA's with unity and $\mathscr{G}, \mathscr{G}^{\prime}$ the HSGLA's coming from $\mathscr{A}, \mathscr{A}^{\prime}$ respectively. Then the following four statements are equivalent to one another:

(a) $\mathscr{A}$ is equivalent to $\mathscr{A}^{\prime}$.

(b) $\mathscr{A}_{\mathrm{deg}}, \mathscr{A}_{\mathrm{non}}$ are equivalent to $\mathscr{A}_{\mathrm{deg}}^{\prime}, \mathscr{A}_{\mathrm{non}}^{\prime}$ respectively.

(c) $\mathscr{G}$ is equivalent to $\mathscr{G}^{\prime}$.

(d) $\mathscr{G}_{\mathrm{deg}}, \mathscr{G}_{\text {non }}$ are equivalent to $\mathscr{G}_{\mathrm{deg}}^{\prime}, \mathscr{G}_{\text {non }}^{\prime}$ respectively.

Proof. (1) The OJTS $\mathscr{V}(\mathscr{A})$ is decomposed into the sum $\mathscr{V}(\mathscr{A})_{\operatorname{deg}} \oplus$ $\mathscr{V}(\mathscr{A})_{\text {non }}$ by Proposition 7.3, (1). Note that the Jordan product on $A$ is given by $X \cdot Y=\{X, Y, E\}_{A}$. Then the objects $\mathscr{A}_{\text {deg }}, \mathscr{A}_{\text {non }}$ are OJA's and the OJA $\mathscr{A}$ is decomposed into the sum $\mathscr{A}_{\mathrm{deg}} \oplus \mathscr{A}_{\text {non }}$. Since $\mathscr{A}$ has the unity, $\mathscr{A}_{\mathrm{deg}}, \mathscr{A}_{\text {non }}$ also have the unities by Proposition 7.1, (2), (a).

(2) Note that $\{X, Y, Z\}_{A}=T_{X \cdot Y}(Z)+\left[T_{X}, T_{Y}\right](Z)$ for $X, Y, Z \in A$. Then our claim is obvious by a routine way.

(3) We show that (a) $\Rightarrow$ (b). Assume that $\mathscr{A}=(A,\langle\rangle)$ is equivalent to $\mathscr{A}^{\prime}=\left(A^{\prime},\langle\rangle^{\prime}\right)$ by an isomorphism $\alpha: \mathscr{A} \widetilde{\rightarrow} \mathscr{A}^{\prime}$. The isomorphism $\alpha$ induces the isomorphism $g_{\alpha}$ of the OJTS $\mathscr{V}(\mathscr{A})$ onto the OJTS $\mathscr{V}\left(\mathscr{A}^{\prime}\right)$. Then $g_{\alpha}$ translates subspaces $\left(V_{A}\right)_{\mathrm{deg}},\left(V_{A}\right)_{\mathrm{non}}$ to subspaces $\left(V_{A^{\prime}}\right)_{\mathrm{deg}},\left(V_{A^{\prime}}\right)_{\mathrm{non}}$ respectively. Hence $\alpha$ translates JA's $A_{\text {deg }}, A_{\text {non }}$ to JA's $A_{\text {deg }}^{\prime}, A_{\text {non }}^{\prime}$ respectively. This implies that $\mathscr{A}_{\text {deg }}, \mathscr{A}_{\text {non }}$ are equivalent to $\mathscr{A}_{\text {deg }}^{\prime}, \mathscr{A}_{\text {non }}^{\prime}$ respectively.

The claims that $(b) \Rightarrow(d),(c) \Rightarrow$ (a) are obvious by Theorem $5.5,(2)$ and the claim that (d) $\Rightarrow$ (c) by the claim (1).

Let $\mathscr{A}$ be an OJA with unity and $\mathscr{G}$ the HSGLA coming from $\mathscr{A}$. Denote the OJA $\mathscr{A}_{\text {non }}$, the HSGLA $\mathscr{G}_{\text {non }}$ by $\mathscr{A}_{\text {non }}=\left(A_{\text {non }},\langle\rangle_{\text {non }}\right), \mathscr{G}_{\text {non }}=$ $\left(\mathfrak{g}_{\text {non }}=\sum\left(\mathfrak{g}_{\text {non }}\right)_{\mu}, \rho_{\text {non }}, J_{p_{\text {non }}},\langle\rangle_{\text {pnon }_{n}}\right)$. Then the Lie algebra $\mathfrak{g}_{\text {non }}$ is semi-simple by Propositions 7.3, (3) and 7.4, (2). Let $\mathfrak{g}_{\mathrm{non}}=\mathfrak{g}_{1} \oplus \ldots \oplus \mathfrak{g}_{s}$ be the decom- 
position of the Lie algebra $\mathfrak{g}_{\text {non }}$ into simple Lie algebras $\mathfrak{g}_{i}$. Define subspaces $A_{i}, 1 \leqq i \leqq s$, of $A_{\text {non }}$ by $A_{i}=\left\{X \in A_{\text {non }} ;(X, 0,0) \in \mathfrak{g}_{i}\right\}$ and denote by \langle\rangle$_{i}, 1 \leqq i \leqq s$, the restrictions of \langle\rangle$_{\text {non }}$ into subspaces $A_{i}$ respectively.

TheOREm 7.5. (1) The objects $\mathscr{A}_{i}=\left(A_{i},\langle\rangle_{i}\right), 1 \leqq i \leqq s$, are OJA's with unity and the OJA $\mathscr{A}_{\text {non }}$ is decomposed into the sum $\oplus \mathscr{A}_{i}$. Hence the OJA $\mathscr{A}$ is decomposed into the sum $\mathscr{A}_{\mathrm{deg}} \oplus\left(\oplus \mathscr{A}_{i}\right)$.

(2) Let $\mathscr{G}_{i}, 1 \leqq i \leqq s$, be the HSGLA's coming from $\mathscr{A}_{i}$ respectively. Then the Lie algebras underlying $\mathscr{G}_{i}, 1 \leqq i \leqq s$, are isomorphic to $\mathfrak{g}_{i}$ respectively and the HSGLA $\mathscr{G}_{\text {non }}$ is equivalent to the sum $\oplus \mathscr{G}_{i}$. Hence the HSGLA $\mathscr{G}$ is equivalent to the sum $\mathscr{G}_{\mathrm{deg}} \oplus\left(\oplus \mathscr{G}_{i}\right)$.

(3) Let $\mathscr{A}, \mathscr{A}^{\prime}$ be OJA's with unity and $\mathscr{G}, \mathscr{G}^{\prime}$ the HSGLA's coming from $\mathscr{A}, \mathscr{A}^{\prime}$ respectively. Let $\mathscr{A}_{\mathrm{deg}} \oplus\left(\oplus_{i=1}^{s} \mathscr{A}_{i}\right), \mathscr{A}_{\mathrm{deg}}^{\prime} \oplus\left(\oplus_{i=1}^{t} \mathscr{A}_{i}^{\prime}\right)$ be the decompositions of $\mathscr{A}, \mathscr{A}^{\prime}$ respectively given in (1) and let $\mathscr{G}_{\mathrm{deg}} \oplus\left(\oplus_{i=1}^{s} \mathscr{G}_{i}\right)$, $\mathscr{G}_{\mathrm{deg}}^{\prime} \oplus\left(\oplus_{i=1}^{t} \mathscr{G}_{i}^{\prime}\right)$ be the decompositions of $\mathscr{G}, \mathscr{G}^{\prime}$ respectively given in (2). Then the following four statements are equivalent to one another:

(a) $\mathscr{A}$ is equivalent to $\mathscr{A}^{\prime}$.

(b) The object $\left(\mathscr{A}_{\mathrm{deg}}, \mathscr{A}_{1}, \cdots, \mathscr{A}_{s}\right)$ is equivalent to the object $\left(\mathscr{A}_{\mathrm{deg}}^{\prime}, \mathscr{A}_{1}^{\prime}\right.$, $\cdots, \mathscr{A}_{t}^{\prime}$ ), i.e., (i) $\mathscr{A}_{\mathrm{deg}}$ is equivalent to $\mathscr{A}_{\mathrm{deg}}^{\prime}$, and (ii) $s=t$ and there exists a permutation $\Sigma$ such that $\mathscr{A}_{\Sigma(i)}^{\prime}$ is equivalent to $\mathscr{A}_{i}$ for any $i$.

(c) $\mathscr{G}$ is equivalent to $\mathscr{G}^{\prime}$.

(d) The object $\left(\mathscr{G}_{\mathrm{deg}}, \mathscr{G}_{1}, \ldots, \mathscr{G}_{s}\right)$ is equivalent to the object ( $\mathscr{G}_{\mathrm{deg}}^{\prime}, \mathscr{G}_{1}^{\prime}$, $\ldots, \mathscr{G}_{t}^{\prime}$ ), i.e., (i) $\mathscr{G}_{\mathrm{deg}}$ is equivalent to $\mathscr{G}_{\mathrm{deg}}^{\prime}$, and (ii) $s=t$ and there exists $a$ permutation $\Sigma$ such that $\mathscr{G}_{\Sigma(i)}^{\prime}$ is equivalent to $\mathscr{G}_{i}$ for any $i$.

Proof. (1) Let $\nu=\left(0,-\mathrm{id}_{A_{\mathrm{non}}}, 0\right) \in\left(\mathrm{g}_{\mathrm{non}}\right)_{0}$. Then $\left(\mathrm{g}_{\mathrm{non}}\right)_{\mu}, \mu=0, \pm 1$, are characterized as eigen spaces of ad( $\nu$ ) for eigen values $\mu$ respectively. Hence, putting $\left(\mathfrak{g}_{i}\right)_{\mu}=\mathfrak{g}_{i} \cap\left(\mathfrak{g}_{\text {non }}\right)_{\mu}, \mu=0, \pm 1,1 \leqq i \leqq s$, we have

$$
\mathfrak{g}_{i}=\sum_{\mu}\left(\mathfrak{g}_{i}\right)_{\mu}
$$

for $1 \leqq i \leqq s$ and

$$
\left(\mathfrak{g}_{\mathrm{non}}\right)_{\mu}=\bigoplus_{i=1}^{s}\left(\mathfrak{g}_{i}\right)_{\mu}
$$

for $\mu=0, \pm 1$. Since $\left(\mathfrak{g}_{\mathrm{non}}\right)_{-1}=\left(A_{\text {non }}, 0,0\right)$ and $\left(\mathfrak{g}_{i}\right)_{-1}=\left(A_{i}, 0,0\right), 1 \leqq i \leqq s$, the JA $A_{\text {non }}$ is decomposed into the sum $\oplus A_{i}$ of linear subspaces $A_{i}$ by (7.7). Let $X \in A_{i}, Y \in A_{\text {non }}$. Since $g_{i}$ is an ideal of $g_{\text {non }}$, we have

$$
(X \cdot Y, 0,0)=\left[\left(0, T_{Y}, 0\right),(X, 0,0)\right] \in \mathfrak{g}_{i}
$$

and thus $X \cdot Y \in A_{i}$. This implies that $A_{i}$ is an ideal of $A$. Hence the 
JA $A_{\text {non }}$ is decomposed into the sum $\oplus A_{i}$ of JA's $A_{i}$.

Let $\mathscr{V}\left(\mathscr{A}_{\text {non }}\right)=\left(V_{\text {non }},\{\}_{\text {non }},\langle\rangle_{\text {non }}\right)$. Regard $A_{i}, 1 \leqq i \leqq s$, as subspaces $V_{i}$ of $V_{\text {non }}$ respectively and denote by \{\}$_{i}, 1 \leqq i \leqq s$, the restrictions of \{\}$_{\text {non }}$ into subspaces $V_{i}$ respectively. Then the JTS ( $V_{\text {non }},\{\}_{\text {non }}$ ) is decomposed into the sum $\oplus\left(V_{i},\{\}_{i}\right)$ of JTS's $\left(V_{i},\{\}_{i}\right)$ and moreover the symmetric endomorphism $L_{\curlyvee\left(x_{n}\right)}$ is non-degenerate on $V_{\text {non }}$ and thus on $V_{i}, 1 \leqq i \leqq s$, by Proposition 7.3, (2), (b). Hence the restrictions \langle\rangle$_{i}$, $1 \leqq i \leqq s$, of \langle\rangle$_{\text {non }}$ into subspaces $V_{i}$ are non-degenerate by Lemma 7.2. This implies that the OJA $\mathscr{A}_{\text {non }}$ is decomposed into the sum $\oplus \mathscr{A}_{i}$ of OJA's $\mathscr{A}_{i}$. Since $\mathscr{A}_{\text {non }}$ has the unity, $\mathscr{A}_{i}, 1 \leqq i \leqq s$, also have the unities.

(2) Let $E_{\text {non }}$ be the unity of $\mathscr{A}_{\text {non }}$ and put $\varepsilon_{-1}=\left(E_{\text {non }}, 0,0\right) \in\left(\mathfrak{g}_{\text {non }}\right)_{-1}$, $\boldsymbol{\varepsilon}_{1}=\left(0,0, E_{\text {non }}\right) \in\left(g_{\text {non }}\right)_{1}$. Then we have

$$
\begin{aligned}
& \operatorname{ad}\left(\varepsilon_{-1}\right)^{2}(0,0, X)=(1 / 2)(X, 0,0), \\
& \operatorname{ad}\left(\varepsilon_{1}\right)^{2}(X, 0,0)=-(1 / 2)(0,0, X)
\end{aligned}
$$

for $X \in A_{\text {non. }}$. This implies that $(X, 0,0) \in\left(g_{i}\right)_{-1}$ if and only if $(0,0, X) \in\left(g_{i}\right)_{1}$. Since $\left(\mathrm{g}_{i}\right)_{-1}=\left(A_{i}, 0,0\right)$ by definition, we have $\left(\mathrm{g}_{i}\right)_{1}=\left(0,0, A_{i}\right)$, and thus $\left(\mathrm{g}_{i}\right)_{0}=\left\{\left(0, L_{\text {non }}(Z, W), 0\right) ; Z, W \in A_{i}\right\}$ by (7.6). Note that $L_{i}(Z, W), Z, W \in A_{i}$, are the restrictions of $L_{\text {non }}(Z, W)$ into $A_{i}$. Define a mapping $\lambda_{i}$ of the Lie algebra underlying $\mathscr{G}_{i}$ onto the Lie algebra $\mathfrak{g}_{i}$ by

$$
\lambda_{i}\left(X, L_{i}(Z, W), Y\right)=\left(X, L_{\text {non }}(Z, W), Y\right)
$$

for $X, Y, Z, W \in A_{i}$. Then we can easily see that $\lambda_{i}$ is an isomorphism. Hence the Lie algebras underlying $\mathscr{G}_{i}, 1 \leqq i \leqq s$, are isomorphic to the Lie algebras $\mathfrak{g}_{i}$ respectively.

The other claim is obvious by (1).

(3) The claim that (c) $\Rightarrow$ (d) is ovbious by Proposition 7.4, (3) and Shur's Lemma, the claim that $(b) \Rightarrow(a)$ by $(1)$, and the claims that $(d) \Rightarrow$ (b), (a) $\Rightarrow$ (c) by Theorem 5.5, (2).

q.e.d.

Remark 7.6. Let $\mathscr{G}, \mathscr{G}_{\mathrm{deg}}, \mathscr{G}_{i}, 1 \leqq i \leqq s$, be as given in (2) and $\hat{M}_{\mathfrak{g}}^{n} \subset$ $\boldsymbol{H}_{\mathscr{s}}^{n}, \hat{M}_{\mathfrak{s}_{\mathrm{deg}}}^{n_{0}} \subset \boldsymbol{H}_{\vartheta_{\mathrm{deg}}}^{n_{0}}, \hat{M}_{\mathfrak{s}_{i}}^{n_{i}} \subset \boldsymbol{H}_{\vartheta_{i}}^{n_{i}}, 1 \leqq i \leqq s$, the complete totally real parallel submanifolds associated with $\mathscr{G}, \mathscr{G}_{\mathrm{deg}}, \mathscr{G}_{i}$ respectively. Then $\hat{M}_{\mathscr{g}}^{n} \subset \boldsymbol{H}_{\mathscr{g}}^{n}$ is holomorphically and linearly congruent to the product:

$$
\hat{M}_{\mathscr{q}_{\mathrm{deg}}}^{n_{0}} \times \hat{M}_{\mathscr{\vartheta}_{1}}^{n_{1}} \times \cdots \times \hat{M}_{\mathscr{s}_{s}}^{n_{s}} \subset \boldsymbol{H}_{\mathscr{s}_{\mathrm{deg}}}^{n_{0}} \times \boldsymbol{H}_{\mathscr{G}_{1}}^{n_{1}} \times \cdots \times \boldsymbol{H}_{\mathscr{s}_{s}}^{n_{s}}
$$

Remark 7.7. We give a geometric view for the symmetric endomorphism $L_{r(\infty)}$, which plays an important role for the decompositions of $\mathscr{A}$, 
G. Define an element $\eta_{\star} \in A$ by $\eta_{\star}=n \cdot L_{\varkappa(*)}(E)$. Let $\left\{e_{1}, \cdots, e_{k}, e_{k+1}, \cdots, e_{n}\right\}$ be an orthonormal basis of $A$. Then we have

$$
n \cdot \eta_{\mathscr{A}}=-\sum_{i=1}^{k} e_{i} \cdot e_{i}+\sum_{j=k+1}^{n} e_{j} \cdot e_{j}
$$

and thus

$$
n \cdot T_{\eta \mathscr{A}}=L_{r(\mathscr{A})}
$$

by (7.1). Put $\eta_{\star}=\left(\eta_{\star}, 0,-\eta_{\star}\right) \in \mathfrak{p}_{A}$. Then the vector $-J_{p_{A}}\left(\eta_{\star}\right)$ is the mean curvature vector at $\nu$ of the complete totally real parallel submanifold $\hat{M}_{\mathscr{g}}^{n} \subset \boldsymbol{H}_{\mathscr{g}}^{n}$ by (6.11).

Now we apply Theorem 7.5 for the classification of $r$-dimensional complete parallel submanifolds of $\bar{M}^{r}(c), c \neq 0$.

LEMma 7.8. Let $\mathscr{A}$ be an OJA with unity and $\mathscr{G}$ the HSGLA coming from $\mathscr{A}$. Assume that the symmetric bilinear form underlying $\mathscr{A}$ is positive definite. Then the Lie algebra underlying $\mathscr{G}$ is semi-simple.

Proof. Note that the pseudo-Hermitian space $\boldsymbol{H}_{s}^{n}$ is isometric to the Euclidean space $R^{2 n}$ and thus that the parallel submanifold $\hat{M}_{\mathscr{s}}^{n}$ is contained in a sphere of $R^{2 n}$. Hence the Lie algebra underlying the HSGLA $\mathscr{G}_{\mathbb{H}_{\mathcal{G}}}$ associated with $\hat{M}_{\mathscr{Y}}^{n}$ is semi-simple (Ferus [3], Takeuchi [12]). This implies that the Lie algebra underlying $\mathscr{G}$ is semi-simple, since $\mathscr{G}$ is equivalent to $\mathscr{G}_{\hat{\mathbb{H}}_{\mathcal{G}}}$ by Remark 6.5. q.e.d.

In our categories, an object is called decomposable if it is decomposed into the sum of two proper objects, and is called indecomposable if not so.

Lemma 7.9. Let $\mathscr{A}=(A,\langle\rangle)$ be an OJA with unity and $\mathscr{G}=$ $\left(\mathfrak{g}_{A}=\sum\left(\mathfrak{g}_{A}\right)_{\mu}, \rho_{A}, J_{\mathfrak{p} A},\langle\rangle_{\mathfrak{p}_{A}}\right)$ the HGSLA coming from $\mathscr{A}$. Then,

(1) the following five objects are indecomposable if one of them is so: OJA $\mathscr{A}, H S G L A \mathscr{G}$, OJTS $\mathscr{V}(\mathscr{A})$, OSGLA O(G), OSLA underlying $\mathscr{G}$. Moreover,

(2) $\mathscr{G}$ is indecomposable if either of the following conditions is satisfied:

(a) The Lie algebra underlying $\mathscr{G}$ is simple.

(b) $L_{r(\infty)}^{\ell}=0$ for some $\ell$ and the signature of $\langle>$ is $(1, n-1)$.

Proof. (1) It is obvious by Propsition 7.1, (1) (resp. Proposition 7.1, (2)) that $\mathscr{V}(\mathscr{A})$ (resp. $\mathscr{A}$ ) is indecomposable if and only if $\mathscr{O}(\mathscr{G})$ (resp. $\mathscr{G}$ ) is so. Note that the complex structure $J_{p_{A}}$ is given by $J_{p_{A}}=\operatorname{ad}(J) \mid \mathfrak{p}_{A}$ and 
that the Lie subalgebras $\left(\mathfrak{g}_{A}\right)_{\mu}$ of $\mathfrak{g}_{A}$ are characterized as eigen spaces of ad (ע) for eigen values $\mu$ respectively. Then it is obvious that $\mathscr{G}$ is indecomposable if and only if $\mathcal{O}(\mathscr{G})$ is so if and only if the OSLA underlying $\mathscr{G}$ is so.

(2) It is obvious that $\mathscr{G}$ is indecomposable if (a). We show that $\mathscr{G}$ is indecomposable if (b). Assume that $\mathscr{G}$ is decomposed into the sum $\mathscr{G}_{1} \oplus \mathscr{G}_{2}$ of proper HSGLA's $\mathscr{G}_{i}$. Then we may assume that $\mathscr{G}_{i}, i=1,2$, come from OJA's $\mathscr{A}_{i}=\left(A_{i},\langle\rangle_{i}\right)$ with unity respectively, such that $\mathscr{A}$ is equivalent to the sum $\mathscr{A}_{1} \oplus \mathscr{A}_{2}$ by Proposition 7.1, (2), (b) and (3). Since the signature of \langle\rangle is $(1, n-1)$, either of \langle\rangle$_{i}, i=1,2$, is positive definite. Assume that \langle\rangle$_{1}$ is so. Then the Lie algebra underlying $\mathscr{G}_{1}$ is semisimple by Lemma 7.8. Hence $L_{\Upsilon\left(\alpha_{1}\right)}$ is non-degenerate on $A_{1}$ by Theorem 5.4, (4). This contradicts that $L_{\mathscr{r}(\mathscr{A})}^{\ell}=0$. Hence $\mathscr{G}$ is indecomposable.

q.e.d.

Denote by $\mathscr{A}_{c}$ an OJA with unity satisfying the conditions $\left(\mathrm{E}_{c} 1\right)$, $\left(\mathrm{E}_{c} 2\right)$ and $\mathscr{G}_{\mathrm{c}}$ the HSGLA coming from $\mathscr{A}_{c}$. Then, by Lemma 7.9 and Theorem 7.5, (2), an HSGLA $\mathscr{G}_{c}$ is indecomposable if and only if it satisfies either of the following:

(a) The Lie algebra underlying $\mathscr{G}_{c}$ is simple.

(b) $L_{r\left(\mathscr{A}_{c}\right)}^{\ell}=0$ for some $\ell$.

The HSGLA $\mathscr{G}_{c}$ is called simple if it satisfies (a) and is called almost nilpotent if it satisfies (b). Note that $c<0$ for an almost nilpotent HSGLA $\mathscr{G}_{c}$.

Fix a real number $c \neq 0$ and an integer $r \geqq 0$. Let $\mathscr{J}=\left(\left(\mathscr{G}_{0}\right)_{c}, \cdots\right.$, $\left.\left(\mathscr{G}_{s}\right)_{c_{s}}\right)$ be an object consisting of indecomposable HSGLA's $\left(\mathscr{G}_{i}\right)_{c_{i}}, 0 \leqq i \leqq s$, such that (i) $\sum_{i=0}^{s} 1 / c_{i}=1 / c$, and (ii) the signature of the symmetric bilinear form underlying $\oplus_{i=0}^{s}\left(\mathscr{A}_{i}\right)_{c_{i}}$ is $(1, r)$ or $(0, r+1)$ according as $c<0$ or $c>0$. Two objects $\mathscr{J}=\left(\left(\mathscr{G}_{0}\right)_{c 0}, \cdots,\left(\mathscr{G}_{s}\right)_{c_{s}}\right), \mathscr{J}^{\prime}=\left(\left(\mathscr{G}_{0}^{\prime}\right)_{c_{0}^{\prime}}, \cdots,\left(\mathscr{G}_{t}^{\prime}\right)_{c_{t}^{\prime}}\right)$ satisfying the conditions (i), (ii), are said to be equivalent to each other if $s=t$ and there exists a permutation $\Sigma$ such that $\left(\mathscr{G}_{\Sigma(i)}^{\prime}\right)_{c^{\prime}(i)}$ is equivalent to $\left(\mathscr{G}_{i}\right)_{c_{i}}$ for any $i$. Here we note that $c_{\Sigma(i)}^{\prime}=c_{i}$ for any $i$.

Now let us define an object $\mathscr{J}_{M}$ associated with an $r$-dimensional complete totally real parallel submanifold $M^{r}$ of $\bar{M}^{r}(c)$. Let $\mathscr{A}_{\hat{M}}$ (resp. $\mathscr{G}_{\hat{M}}$ ) be the OJA (resp. HSGLA) associated with the complete inverse $\hat{M}^{r+1}$ of $M^{r}$, and $\left(\mathscr{A}_{\hat{K}}\right)_{\mathrm{deg}} \oplus\left(\oplus_{i=1}^{s}\left(\mathscr{A}_{\hat{H}}\right)_{i}\right)$ (resp. $\left.\left(\mathscr{G}_{\hat{M}}\right)_{\mathrm{deg}} \oplus\left(\oplus_{i=1}^{s}\left(\mathscr{G}_{\hat{M}}\right)_{i}\right)\right)$ the decomposition of $\mathscr{A}_{\hat{M}}$ (resp. $\mathscr{G}_{\hat{H}}$ ) given in Theorem 7.5. Denote by $E_{\mathrm{deg}}, E_{i}, 1 \leqq i \leqq s$, the unities of $\left(\mathscr{A}_{\mathscr{M}}\right)_{\mathrm{deg}},\left(\mathscr{A}_{\mathscr{M}}\right)_{i}$ respectively and by \langle\rangle$_{\mathrm{deg}},\langle\rangle_{i}, 1 \leqq i \leqq s$, the 
non-degenerate symmetric bilinear forms underlying $\left(\mathscr{A}_{\mathscr{M}}\right)_{\mathrm{deg}},\left(\mathscr{A}_{\mathscr{A}}\right)_{i}$ respectively. Define real numbers $c_{i}, 0 \leqq i \leqq s$, by $\left\langle E_{\mathrm{deg}}, E_{\mathrm{deg}}\right\rangle_{\mathrm{deg}}=4 / c_{0}$, $\left\langle E_{i}, E_{i}\right\rangle_{i}=4 / c_{i}$. Then, since $\mathscr{A}_{\hat{M}}$ satisfies the conditions $\left(E_{c} 1\right),\left(E_{c} 2\right)$ by Lemma 6.2 , OJA's $\left(\mathscr{A}_{\mathscr{A}}\right)_{\mathrm{deg}}$ and $\left(\mathscr{A}_{\mathscr{A}}\right)_{i}, 1 \leqq i \leqq s$, satisfy the conditions $\left(\mathrm{E}_{c_{0}} 1\right),\left(\mathrm{E}_{c_{0}} 2\right)$ and $\left(\mathrm{E}_{c_{i}} 1\right),\left(\mathrm{E}_{c_{i}} 2\right)$ respectively, and moreover the object $\left(\left(\mathscr{G}_{\hat{M}}\right)_{\mathrm{deg}},\left(\mathscr{G}_{\hat{M A}}\right)_{1}, \cdots,\left(\mathscr{G}_{\hat{B}}\right)_{s}\right)$ satisfies the conditions (i), (ii). We denote this object by $\mathscr{J}_{M}$.

Conversely, let $\mathscr{J}=\left(\left(\mathscr{G}_{0}\right)_{c_{0}}, \cdots,\left(\mathscr{G}_{s}\right)_{c_{s}}\right)$ be an object satisfying the conditions (i), (ii) and put $\mathscr{G}_{c}=\oplus_{i=0}^{s}\left(\mathscr{G}_{i}\right)_{c_{i}}$. Then $\mathscr{G}_{c}$ is the HSGLA coming from an OJA $\mathscr{A}_{c}$ with unity satisfying the conditions $\left(\mathrm{E}_{c} 1\right),\left(\mathrm{E}_{c} 2\right)$. For simplicity, denote by $M_{\xi}^{r} \subset \bar{M}_{\xi}^{r}(c)$ the complete totally real parallel submanifold $M_{\mathscr{s}_{c}}^{r} \subset \bar{M}_{\mathscr{s}_{c}}^{r}(c)$ associated with the HSGLA $\mathscr{G}_{c}$.

THEOREM 7.10. (1) Let $M^{r}$ be an r-dimensional complete totally real parallel submanifold of $\bar{M}^{r}(c), c \neq 0$. Then $M_{g_{M}}^{r} \subset \bar{M}_{\xi_{M}}^{r}(c)$ is holomorphically congruent to $M^{r} \subset \bar{M}^{r}(c)$.

(2) Let $\mathscr{J}$ is an object satisfying the conditions (i), (ii). Then $\mathscr{J}_{M_{g}^{r}}$ is equivalent to $\mathscr{J}$.

(3) Let $\mathscr{J}, \mathscr{J}^{\prime}$ be objects satisfying the conditions (i), (ii). Then $\mathscr{J}$ is equivalent to $\mathscr{J}^{\prime}$ if and only if $M_{\mathscr{g}}^{r} \subset \bar{M}_{\mathscr{g}}^{r}(c)$ is holomorphically congruent to $M_{g^{\prime}}^{r} \subset \bar{M}_{g^{\prime}}^{r}(c)$.

Proof. The claims (1), (2) are obvious by Theorem 6.3, (1), (2) respectively and the claim (3) by Theorem 6.3, (3) and Theorem 7.5, (3).

q.e.d.

Remark 7.11. Let $\mathscr{J}=\left(\left(\mathscr{G}_{0}\right)_{c_{0}}, \cdots,\left(\mathscr{G}_{s}\right)_{c_{s}}\right)$ be an object satisfying the conditions (i), (ii). Assume that $c>0$. Then all $\left(\mathscr{G}_{i}\right)_{c_{i}}$ are simple and all $c_{i}$ are positive. Hence Theorem 7.10 is a reproduction of Theorem 3.1.

Assume that $c<0$. Then there exists an index $i$ such that $\left(\mathscr{G}_{i}\right)_{c_{i}}$ is simple or almost nilpotent with $c_{i}<0$ and that $\left(\mathscr{G}_{j}\right)_{c_{j}}, j \neq i$, are simple with $c_{j}>0$.

\section{§ 8. Almost nilpotent HSGLA's $\mathscr{G}_{c}$}

Let $\mathscr{A}_{c}$ be an OJA with unity satisfying the conditions $\left(\mathrm{E}_{c} 1\right),\left(\mathrm{E}_{c} 2\right)$ and $\mathscr{G}_{c}$ the HSGLA coming from $\mathscr{A}_{c}$. If $\mathscr{G}_{c}$ is almost nilpotent, the Lie algebra underlying $\mathscr{G}_{c}$ is neither semi-simple nor solvable by Proposition 7.3 , (3), the condition (SGL 4 ) and the property that id $\in\left(\mathfrak{g}_{c}\right)_{0}$.

Now Cahen-Parker [2] has studied indecomposable effective OSLA's 
and HSLA's such that the Lie algebras underlying them are neither semisimple nor solvable. We pick up some results which we need in this section.

Let $\left(\mathfrak{g}, \rho,\langle\rangle_{\mathfrak{p}}\right.$ ) be an OSLA. If the OSLA is effective and satisfies that $\mathfrak{f}=[\mathfrak{p}, \mathfrak{p}]$, the form \langle\rangle$_{\mathfrak{p}}$ on $\mathfrak{p}$ is uniquely extended into a non-degenerate symmetric bilinear form on $\mathfrak{g}$ which is left invariant by $\rho$ and which ad $(T), T \in \mathfrak{g}$, are skew symmetric for. We denote by $\langle>$ the bilinear form on $g$.

Let $(\mathfrak{g}, \rho)$ be an SLA. A subspace $\mathfrak{g}$ of $\mathfrak{g}$ is called $\rho$-invariant if $\rho(\mathfrak{g})$ $=\mathfrak{B}$. A $\rho$-invariant subspace $\mathfrak{B}$ is decomposed into the sum of $\mathfrak{g} \cap \mathfrak{f}, \mathfrak{g} \cap \mathfrak{p}$. These subspaces $\mathfrak{I} \cap \mathfrak{f}, \mathfrak{Z} \cap \mathfrak{p}$ are denoted by $\mathfrak{g}_{k}, \mathfrak{z}_{p}$ respectively. $A$ Levi decomposition of $\mathrm{g}$ into the sum of radical $\mathscr{R}$ and semi-simple subalgebra $\mathscr{S}$ is called $\rho$-invariant if $\rho(\mathscr{S})=\mathscr{S}$. There always exists a $\rho$-invariant Levi decomposition of $\mathfrak{g}$.

Lemma 8.1 (Cahen-Parker [2]). Let $\mathscr{G}=\left(\mathfrak{g}, \rho,\langle\rangle_{\mathrm{p}}\right)$ be an indecomposable effective OSLA satisfying that

( 0 ) $\mathfrak{i}=[\mathfrak{p}, \mathfrak{p}]$,

(i) $g$ is neither semi-simple nor solvable,

(ii) $\mathscr{G}$ underlies an HSLA,

(iii) the signature of \langle\rangle$_{\mathfrak{p}}$ is $(2,2 r), r \geqq 1$.

Let $\mathrm{g}=\mathscr{R} \oplus \mathscr{S}$ be a $\rho$-invariant Levi decomposition. Then,

(1) the radical $\mathscr{R}$ is nilpotent and $\operatorname{dim} \mathscr{S}=3$,

(2) $C_{\mathfrak{t}}(\mathscr{S})=\{X \in \mathfrak{F} ;[X, \mathscr{S}]=\{0\}\}=\{0\}$.

Moreover,

(3) the nilpotent radical $\mathscr{R}$ is decomposed into the sum $\mathscr{V} \oplus \mathscr{S}^{\prime}$ of mutually orthogonal $\rho$-invariant $\mathscr{S}$-modules $\mathscr{V}, \mathscr{S}^{\prime}$ such that

(a) $\mathscr{S}^{\prime}$ is the center of $\mathscr{R}$ and $\operatorname{dim} \mathscr{S}^{\prime}=3$,

(b) $\mathscr{V}$ is orthogonal to $\mathscr{S}$,

(c) $[\mathscr{V}, \mathscr{V}] \subset \mathscr{S}^{\prime}$,

(d) $\left\langle\left[\mathscr{S}_{\mathfrak{p}}, \mathscr{S}_{\mathfrak{p}}^{\prime}\right], \mathscr{R}_{\mathfrak{t}}\right\rangle=\{0\}$.

Hereafter in this section we denote by $\mathscr{G}_{c}=\left(\mathfrak{g}=\sum \mathfrak{g}_{\mu}, \rho, J_{\mathfrak{p}},\langle\rangle_{\mathrm{p}}\right)$ the HSGLA coming from an OJA $\mathscr{A}_{c}=(A,\langle\rangle)$ with the unity $E$ satisfying the conditions $\left(\mathrm{E}_{c} 1\right),\left(\mathrm{E}_{c} 2\right)$, and assume that $\mathscr{G}_{c}$ is almost nilpotent. Then we note that $\mathfrak{f}=[\mathfrak{p}, \mathfrak{p}]$ and $\operatorname{dim} A=r+1 \geqq 2$. Since $c<0$, the signature of \langle\rangle is $(1, r)$ by $\left(\mathrm{E}_{c} 1\right)$ and thus that of \langle\rangle$_{\mathfrak{p}}$ is $(2,2 r)$. Hence the OSLA $\left(\mathfrak{g}, \rho,\langle\rangle_{\mathfrak{p}}\right)$ underlying $\mathscr{G}_{c}$ satisfies the conditions (0), 
(i), (ii), (iii). We apply Lemma 8.1 to this OSLA. Denote by $\mathscr{R}$ the radical of $g$ and put $\mathscr{S}=\left\{\varepsilon_{-1}=(E, 0,0), \varepsilon_{1}=(0,0, E), \nu=(0,-\mathrm{id}, 0)\right\}_{R}$.

LEMma 8.2. (1) The subspace $\mathscr{S}$ is a $\rho$-invariant Lie subalgebra of $\mathrm{g}$ isomorphic to $\operatorname{sl}(2, R)$ and $\mathrm{g}$ is the direct sum of $\mathscr{R}, \mathscr{S}$, i.e., the direct sum $\mathrm{g}=\mathscr{R} \oplus \mathscr{S}$ is a $\rho$-invariant Levi decomposition.

(2) $\left[T_{X}, T_{Y}\right]=0$ for $X, Y \in A$.

Proof. (1) Put $e_{1}=(E, 0, E), e_{2}=(E, 0,-E), e_{3}=\nu$. Then we have $\left[e_{1}, e_{2}\right]=-e_{3},\left[e_{1}, e_{3}\right]=e_{2},\left[e_{2}, e_{3}\right]=e_{1}$. This implies that $\mathscr{S}$ is a Lie subalgebra of $\mathrm{g}$ isomorphic to $\operatorname{sl}(2, R)$. The claim that $\rho(\mathscr{S})=\mathscr{S}$ is obvious by the definition of $\rho$ and the claim that $\mathfrak{g}=\mathscr{R} \oplus \mathscr{S}$ by the simplicity of $\mathscr{S}$ and Lemma 8.1,(1).

(2) Since $\left(0,\left[T_{X}, T_{Y}\right], 0\right) \in \mathfrak{f}$ and $\left[\left(0,\left[T_{X}, T_{Y}\right], 0\right), \mathscr{S}\right]=\{0\}$, we have $\left(0,\left[T_{X}, T_{Y}\right], 0\right) \in C_{t}(\mathscr{S})$, and thus $\left[T_{X}, T_{Y}\right]=0$ by Lemma $8.1,(2)$. q.e.d.

Let $\mathscr{R}=\mathscr{V} \oplus \mathscr{S}^{\prime}$ be the decomposition of $\mathscr{R}$ given in Lemma 8.1, (3). Put

$$
\begin{aligned}
& A_{\mathscr{S}}=\{E\}_{R}, \quad A_{\mathscr{R}}=\left\{X \in A ; T_{X} \text { is nilpotent. }\right\}, \\
& A_{\mathscr{S}^{\prime}}=\left\{\eta=\eta_{\mathscr{s}_{\mathfrak{c}}}\right\}_{R}, \quad A_{\mathscr{r}}=\left\{X \in A_{\mathscr{R}} ;\langle X, E\rangle=0\right\} .
\end{aligned}
$$

LEMMA 8.3. (1) The subspaces $\mathscr{S}, \mathscr{R}, \mathscr{S}^{\prime}, \mathscr{V}$ are characterized by $A_{\mathscr{S}}$, $A_{\mathfrak{R}}, A_{\mathscr{G}^{\prime}}, A_{\boldsymbol{r}}$ respectively as follows:

$$
\begin{array}{ll}
\mathscr{S}=\left\{\left(X, T_{Y}, Z\right) \in \mathfrak{g} ; X, Y, Z \in A_{\mathscr{S}}\right\}, & \mathscr{R}=\left\{\left(X, T_{Y}, Z\right) \in \mathfrak{g} ; X, Y, Z \in A_{\mathfrak{R}}\right\}, \\
\mathscr{S}^{\prime}=\left\{\left(X, T_{Y}, Z\right) \in \mathfrak{g} ; X, Y, Z \in A_{\mathscr{S}^{\prime}}\right\}, & \mathscr{V}=\left\{\left(X, T_{Y}, Z\right) \in \mathfrak{g} ; X, Y, Z \in A_{\mathscr{r}}\right\} .
\end{array}
$$

(2) $\quad A=A_{\mathscr{S}} \oplus A_{\mathfrak{R}}, A_{\mathscr{R}}=A_{\mathscr{r}} \oplus A_{\mathscr{\varphi}}$ and $\operatorname{dim} A_{\mathscr{S}}=1, \operatorname{dim} A_{\mathscr{R}}=r, \operatorname{dim}$ $A_{r}=r-1, \operatorname{dim} A_{\mathscr{S}^{\prime}}=1$.

Proof. It is obvious by the definition of $\mathscr{S}$ that $\mathscr{S}$ is characterized by $A_{\mathscr{S}}$ as above.

Recall that $\mathrm{g}_{\mu}, \mu=0, \pm 1$, are characterized as eigen spaces of $\operatorname{ad}(\nu)$ for eigen values $\mu$ respectively, and note that $\nu \in \mathscr{S}$. Then, since $\mathscr{S}, \mathscr{R}$, $\mathscr{S}^{\prime}, \mathscr{V}$ are $\mathscr{S}$-modules, we have

$$
\left\{\begin{array}{l}
\mathscr{R}=\sum_{\mu} \mathscr{R} \cap \mathfrak{g}_{\mu}, \quad \mathscr{S}^{\prime}=\sum_{\mu} \mathscr{S}^{\prime} \cap \mathfrak{g}_{\mu}, \quad \mathscr{V}=\sum_{\mu} \mathscr{V} \cap \mathfrak{g}_{\mu}, \\
\mathfrak{g}_{\mu}=\mathscr{S} \cap \mathfrak{g}_{\mu} \oplus \mathscr{R} \cap \mathfrak{g}_{\mu}, \quad \mathscr{R} \cap \mathfrak{g}_{\mu}=\mathscr{S}^{\prime} \cap \mathfrak{g}_{\mu} \oplus \mathscr{V} \cap \mathfrak{g}_{\mu}, \quad \mu=0, \pm 1 .
\end{array}\right.
$$

Note that $g_{0}=\left\{\left(0, T_{X}, 0\right) ; X \in A\right\}$ by Lemma $8.2,(2)$, and that $\operatorname{ad}\left(\varepsilon_{1}\right) \mid g_{-1}$ (resp. ad $\left.\left(\varepsilon_{1}\right) \mid \mathfrak{g}_{0}\right)$ is a linear isomorphism of $\mathfrak{g}_{-1}$ (resp. $\mathfrak{g}_{0}$ ) onto $\mathfrak{g}_{0}$ (resp. $\mathfrak{g}_{1}$ ) given in the following. 


$$
\left\{\begin{array}{l}
\operatorname{ad}\left(\varepsilon_{1}\right)(X, 0,0)=(1 / 2)\left(0, T_{X}, 0\right) \\
\left(\operatorname{resp} \cdot \operatorname{ad}\left(\varepsilon_{1}\right)\left(0, T_{X}, 0\right)=(0,0, X)\right) .
\end{array}\right.
$$

Define subspaces $\tilde{A}_{\mathscr{Q}}, \tilde{A}_{\mathscr{S}}, \tilde{A}_{\mathscr{r}}$ by $\mathscr{R} \cap \mathfrak{g}_{-1}=\left(\tilde{A}_{\mathscr{R}}, 0,0\right), \mathscr{S}^{\prime} \cap \mathrm{g}_{-1}=\left(\tilde{A}_{\mathscr{S}^{\prime}}, 0,0\right)$, $\mathscr{V} \cap \mathfrak{g}_{-1}=\left(\tilde{A}_{\mathscr{r}}, 0,0\right)$. Then, since $\varepsilon_{1} \in \mathscr{S}$, we have

$$
\begin{aligned}
& \mathscr{R}=\left\{\left(X, T_{Y}, Z\right) ; X, Y, Z \in \widetilde{A}_{\mathscr{R}}\right\}, \quad \mathscr{S}^{\prime}=\left\{\left(X, T_{Y}, Z\right) ; X, Y, Z \in \widetilde{A}_{\mathscr{P}^{\prime}}\right\}, \\
& \mathscr{V}=\left\{\left(X, T_{Y}, Z\right) ; X, Y, Z \in \widetilde{A}_{\mathscr{r}}\right\}
\end{aligned}
$$

by (8.1), (8.2). Here note that $\operatorname{dim} \tilde{A}_{\mathscr{R}}=r, \operatorname{dim} \tilde{A}_{\mathscr{S}^{\prime}}=1, \operatorname{dim} \tilde{A}_{\mathscr{r}}=r-1$ since $\operatorname{dim} \mathscr{S}=\operatorname{dim} \mathscr{S}^{\prime}=3$ by Lemma 8.1.

To complete our proof, we may show that $\tilde{A}_{\mathscr{R}}=A_{\mathscr{a}}, \tilde{A}_{\mathscr{S}^{\prime}}=A_{\mathscr{C}^{\prime}}$, $\tilde{A}_{r}=A_{r}$.

Take an element $X \in \tilde{A}_{\mathscr{R}}$. Then we have $\left(0, T_{X}, 0\right) \in \mathscr{R}$. Since $\mathscr{R}$ is a 2-step nilpotent ideal by Lemma 8.1 , we have

$$
\left(T_{X}^{3}(Y), 0,0\right)=\operatorname{ad}\left(\left(0, T_{X}, 0\right)\right)^{3}(Y, 0,0) \in[\mathscr{R},[\mathscr{R}, \mathscr{R}]]=\{0\}
$$

for $Y \in A$ and thus $T_{X}^{3}=0$. This implies that $X \in A_{\mathscr{G}}$. Hence we have $\tilde{A}_{\mathscr{R}} \subset A_{\mathscr{R}}$. Note that $E \notin A_{\mathscr{R}}$ since $T_{E}=\mathrm{id}$ is not nilpotent, and thus that $\operatorname{dim} A_{\mathscr{R}} \leqq r$. Then we have $\widetilde{A}_{\mathscr{R}}=A_{\mathscr{R}}$ since $\operatorname{dim} \tilde{A}_{\mathscr{R}}=r$.

Next we show that $\tilde{A}_{r}=A_{r}$. Note that $\operatorname{dim} A_{r} \leqq r-1$. In fact, we have $\eta \in A_{\mathscr{R}}$ since $(r+1) T_{\eta}=L_{\mathscr{r}\left(s_{c}\right)}$ by (7.9). Moreover we have

$$
\begin{aligned}
\langle\eta, E\rangle & =(1 /(r+1))\left\langle-e_{0} \cdot e_{0}+\sum_{i} e_{i} \cdot e_{i}, E\right\rangle \\
& =(1 /(r+1))\left\{-\left\langle e_{0}, e_{0}\right\rangle+\sum_{i}\left\langle e_{i}, e_{i}\right\rangle\right\}=1
\end{aligned}
$$

by (7.8), where $\left\{e_{0}, e_{1}, \cdots, e_{r}\right\}$ denotes an orthonormal basis of $A$. Hence we have $\eta \notin A_{r}$. This implies that $\operatorname{dim} A_{r} \leqq r-1$. Take an element $X \in \tilde{A}_{\mathscr{r}} \subset \tilde{A}_{\mathscr{R}}=A_{\mathfrak{R}}$. Then we have $(X, 0,-X) \in \mathscr{V}$. Since $\mathscr{V}$ is orthogonal to $\mathscr{S}$ by Lemma $8.1,(3)$, (b), we have

$$
\langle X, E\rangle=\left\langle(X, 0,-X),(E, 0,-E)_{\mathfrak{p}}\right\rangle=0
$$

and thus $X \in A_{r}$. This implies that $\tilde{A}_{r} \subset A_{r}$. Since $\operatorname{dim} \tilde{A}_{r}=r-1$, we have $\tilde{A}_{r}=A_{r}$.

Finally we show that $\tilde{A}_{\mathscr{S}^{\prime}}=A_{\mathscr{G}^{\prime}}$. Note that

$$
\left\langle\tilde{A}_{\mathscr{S}^{\prime}}, A_{\mathfrak{Q}}\right\rangle=\{0\} .
$$

In fact, take $X \in \tilde{A}_{\mathscr{S}^{\prime}}, Y \in A_{\mathfrak{s}}$. Then we have $\left(0, T_{X}, 0\right) \in \mathscr{S}_{\mathfrak{p}}^{\prime},(Y, 0, Y) \in \mathscr{R}_{\mathrm{t}}$. Hence we have 


$$
\begin{aligned}
0 & =\left\langle\left[(E, 0,-E),\left(0, T_{X}, 0\right)\right],(Y, 0, Y)\right\rangle=-\left\langle\left(0, T_{X}, 0\right),[(E, 0,-E),(Y, 0, Y)]\right\rangle \\
& =\left\langle\left(0, T_{X}, 0\right),\left(0, T_{Y}, 0\right)\right\rangle_{\mathfrak{p}}=\langle X, Y\rangle
\end{aligned}
$$

by Lemma 8.1, (3), (d). This implies (8.4). Now, since $\left\langle E, \tilde{A}_{\mathscr{S}^{\prime}}\right\rangle \neq\{0\}$, there exists an element $U \in A$ such that $\langle E, U\rangle=0, E+U \in \tilde{A}_{g^{\prime}}$. Then we have

$$
\begin{gathered}
\left\langle U, A_{r}\right\rangle=\{0\}, \quad\langle U, U\rangle=-(4 / c), \\
U \cdot U=-E-2 U .
\end{gathered}
$$

In fact, we have

$$
\{0\}=\left\langle E+U, A_{r}\right\rangle=\left\langle U, A_{r}\right\rangle
$$

and

$$
0=\langle E+U, E+U\rangle=\langle E, E\rangle+\langle U, U\rangle=4 / c+\langle U, U\rangle
$$

by (8.4). These imply (8.5). Since $\mathscr{S}^{\prime}$ is the center of $\mathscr{R}$ by Lemma 8.1 , (3), (a), we have

$$
((E+U) \cdot(E+U), 0,0)=\left[\left(0, T_{E+U}, 0\right),(E+U, 0,0)\right] \in\left[\mathscr{P}^{\prime}, \mathscr{S}^{\prime}\right]=\{0\}
$$

and thus $(E+U) \cdot(E+U)=0$. This implies (8.6). Put $e_{0}=(\sqrt{-c} / 2) E$, $e_{1}=(\sqrt{-c} / 2) U$ and let $\left\{e_{2}, \cdots, e_{r}\right\}$ be an orthonormal basis of $A_{r}$. Then $\left\{e_{0}, e_{1}, \cdots, e_{r}\right\}$ is an orthonormal basis of $A$ by (8.5). Note that

$$
\left(e_{i} \cdot e_{i}, 0,0\right)=\left[\left(0, T_{e_{i}}, 0\right),\left(e_{i}, 0,0\right)\right] \in[\mathscr{V}, \mathscr{V}] \subset \mathscr{S}^{\prime}
$$

for $i \geqq 2$ by Lemma 8.1,(3), (c), and thus $e_{i} \cdot e_{i} \in \tilde{A}_{\mathcal{S}^{\prime}}, i \geqq 2$. Then we have

$$
\begin{aligned}
(r+1) \eta & =-e_{0} \cdot e_{0}+e_{1} \cdot e_{1}+\sum_{i=2}^{r} e_{i} \cdot e_{i} \\
& =(c / 2)(E+U)+\sum_{i=2}^{r} e_{i} \cdot e_{i} \in \tilde{A}_{\mathscr{\varphi}}
\end{aligned}
$$

by (8.6). This implies that $A_{\mathscr{S}^{\prime}} \subset \tilde{A}_{\mathscr{S}^{\prime}}$. Since $\eta \neq 0$ and $\operatorname{dim} \tilde{A}_{\mathscr{S}^{\prime}}=1$, we have $A_{\mathscr{S}^{\prime}}=\tilde{A}_{\mathscr{S}^{\prime}}$.

q.e.d.

Lemma 8.4. The Jordan product on $A$ and the non-degenerate symmetric bilinear form $\langle>$ on $A$ are given in the following.

$$
\begin{cases}E \cdot X=X \cdot E=X & \text { for } X \in A, \\ \eta \cdot Y=Y \cdot \eta=0 & \text { for } Y \in A_{\mathfrak{x}}, \\ Z \cdot W=W \cdot Z=\langle Z, W\rangle_{\eta} & \text { for } Z, W \in A_{r},\end{cases}
$$

and 


$$
\left\{\begin{array}{l}
\langle E, E\rangle=4 / c,\langle E, \eta\rangle=1,\left\langle E, A_{r}\right\rangle=\{0\}, \\
\langle\eta, \eta\rangle=0,\left\langle\eta, A_{r}\right\rangle=\{0\} .
\end{array}\right.
$$

Proof. Since $\mathscr{S}^{\prime}$ is the center of $\mathscr{R}$, we have

$$
(\eta \cdot Y, 0,0)=\left[\left(0, T_{\eta}, 0\right),(Y, 0,0)\right] \in\left[\mathscr{S}^{\prime}, \mathscr{R}\right]=\{0\}
$$

for $Y \in A_{\mathscr{R}}$ and thus $\eta \cdot Y=0$.

Let $Z, W \in A_{r}$. Since $[\mathscr{V}, \mathscr{V}] \subset \mathscr{S}^{\prime}$, we have

$$
(Z \cdot W, 0,0)=\left[\left(0, T_{Z}, 0\right),(W, 0,0)\right] \in \mathscr{S}^{\prime}
$$

and thus $Z \cdot W \in A_{\mathscr{9}}$. Put $Z \cdot W=\lambda(Z, W) \eta$. Then we have

$$
\langle Z \cdot W, E\rangle=\lambda(Z, W)\langle\eta, E\rangle=\lambda(Z, W)
$$

by (8.3) and thus $\lambda(Z, W)=\langle Z, W\rangle$. This implies that $Z \cdot W=\langle Z, W\rangle \eta$.

The other equations of (8.7) are obvious. In the equations of (8.8) the first is clear by $\left(\mathrm{E}_{c} 2\right)$, the second by (8.3), the third by the definition of $A_{\mathscr{r}}$, the forth and the fifth by (8.4). $\quad$ q.e.d.

Fix an integer $r \geqq 1$ and a negative number $c$. Let $\mathscr{P}=(P,\langle\rangle)$ be an object consisting of $(r-1)$-dimensional real vector space $P$ and positive definite inner product $\langle>$ on $P$. Let $E, \eta$ be elements. Define an $(r+1)$-dimensional real vector space $A$ by $A=R \cdot E \oplus R \cdot \eta \oplus P$. And define a product on $A$ by (8.7) and extend the inner product $\langle>$ on $P$ into a symmetric bilinear form on $A$ by (8.8). This symmetric bilinear form on $A$ will be also denoted by $\langle>$. We denote this object $(A,\langle\rangle)$ by $\mathscr{A}(r, c, \mathscr{P}, E, \eta)$.

TheOREM 8.5. (1) The object $\mathscr{A}(r, c, \mathscr{P}, E, \eta)$ is an OJA with the unity $E$ satisfying the conditions $\left(\mathrm{E}_{c} 1\right),\left(\mathrm{E}_{c} 2\right)$, such that $\eta_{\mathscr{A}(r, c, \mathscr{P}, E, \eta)}=\eta$. Moreover the HSGLA $\mathscr{G}(r, c, \mathscr{P}, E, \eta)$ coming from $\mathscr{A}(r, c, \mathscr{P}, E, \eta)$ is almost nilpotent.

(2) Let $\mathscr{A}(r, c, \mathscr{P}, E, \eta), \mathscr{A}\left(r^{\prime}, c^{\prime}, \mathscr{P}^{\prime}, E^{\prime}, \eta^{\prime}\right)$ be OJA's constructed as above. Then $\mathscr{A}(r, c, \mathscr{P}, E, \eta)$ is equivalent to $\mathscr{A}\left(r^{\prime}, c^{\prime}, \mathscr{P}^{\prime}, E^{\prime}, \eta^{\prime}\right)$ if and only if $r=r^{\prime}$ and $c=c^{\prime}$.

(3) Let $\mathscr{A}_{c}=(A,\langle\rangle)$ be an OJA with the unity $E$ satisfying the conditions $\left(\mathrm{E}_{c} 1\right),\left(\mathrm{E}_{c} 2\right)$. Assume that the HSGLA $\mathscr{G}_{c}$ coming from $\mathscr{A}_{c}$ is almost nilpotent. Then $\mathscr{A}_{c}$ is equivalent to $\mathscr{A}(r, c, \mathscr{P}, E, \eta)$, where $r=\operatorname{dim}$ $A-1$ and $\mathscr{P}=\left(A_{r},\langle>| A_{\curlyvee}\right)$.

(4) Let $\mathscr{G}_{c}$ be as in (3). Then the submanifold $\hat{M}_{\mathfrak{s}_{c}}^{r+1}$ is flat, and thus 
so is the submanifold $M_{s_{c}}^{r}$.

Proof. The claim (1) is proved straightforwardly. The claim (2) is clear from the construction of objects and the claim (3) by Lemma 8.4.

We show the claim (4). Let $\mathscr{A}_{\hat{M}_{\mathfrak{M}_{c}^{r+1}}^{r+1}}$ be the OJA associated with $\hat{M}_{\mathfrak{s}_{c}}^{r+1}$. Then $\mathscr{A}_{\hat{M}_{S_{c}}^{r+1}}$ is equivalent to $\mathscr{A}_{c}$ by Theorem 6.3, (2). Hence the curvature endomorphisms $\hat{R}(X, Y), X, Y \in T_{p}\left(\hat{M}_{\mathfrak{s}_{c}}^{r+1}\right)$, of $\hat{M}_{\mathfrak{s}_{c}}^{r+1}$ are identified with $\left[T_{X}\right.$, $\left.T_{Y}\right], X, Y \in A$. Hence $\hat{M}_{\mathfrak{s}_{c}}^{r+1}$ is flat by Lemma 8.2, (2). q.e.d.

\section{§ 9. Simple HSGLA's $\mathscr{G}_{c}$}

Let $\mathscr{A}_{c}=(A,\langle\rangle)$ be an OJA with the unity $E$ satisfying the conditions $\left(\mathrm{E}_{c} 1\right),\left(\mathrm{E}_{c} 2\right)$ and $\mathscr{G}_{c}=\left(\mathfrak{g}=\sum \mathfrak{g}_{\mu}, \rho, J_{\mathfrak{p}},\langle\rangle_{\mathrm{p}}\right)$ the HSGLA coming from $\mathscr{A}_{c}$. Assume that $\mathscr{G}_{c}$ is simple. Then $\mathfrak{g}$ is a simple Lie algebra of non-compact type.

Assume that $c>0$. Then $\left\langle>\right.$ is positive definite by $\left(E_{c} 1\right)$, and thus so is \langle\rangle$_{p}$. This HSGLA $\mathscr{G}_{c}$ is constructed from an object $(D, c)$ of irreducible symmetric bounded domain $D$ of tube type and positive number $c$ (See Naitoh-Takeuchi [10] for the construction).

In this section we study simple HSGLA's $\mathscr{G}_{c}$ such that $c<0$. Such an HSGLA $\mathscr{G}_{c}$ has the following properties:

(1) $\left(g, \rho, J_{p},\langle\rangle_{p}\right)$ is an HSLA such that the signature of \langle\rangle$_{p}$ is $(2,2 r), r \geqq 0$.

(2) $\mathfrak{g}=\sum \mathfrak{g}_{\mu}$ is a GLA such that $\mathfrak{g}_{\mu}, \mu=0, \pm 1$, are eigen spaces of ad (ע) for eigen values $\mu$ respectively.

(3) $2 \operatorname{dim} g_{-1}=\operatorname{dim} \mathfrak{p}$.

Now Berger [1] has classified SLA's $(\mathfrak{g}, \rho)$ with simple Lie algebra $\mathfrak{g}$ of non-compact type and moreover has pointed out SLA's underlying HSLA's among them. Let $(\mathfrak{g}, \rho)$ be an SLA with simple Lie algebra $g$ and let $\mathfrak{g}=\mathfrak{1} \oplus \mathfrak{p}$ be the canonical decomposition of $\mathfrak{g}$ by the involution $\rho$. Then the restriction $B_{\mathfrak{p}}$ of the Killing form $B$ of $\mathrm{g}$ is a non-degenerate symmetric bilinear form on $\mathfrak{p}$ (See Berger [1]). We list up Lie algebras $\mathfrak{g}$, Lie subalgebras $\mathfrak{f}$, and signatures of $B_{\mathfrak{p}}$ for SLA's $(\mathfrak{g}, \rho)$ underlying HSLA's in the following, (Table I).

Now let $g$ be a simple Lie algebra of non-compact type and $e$ an element of $\mathrm{g}$ such that the eigen values of $\operatorname{ad}(e)$ are $0, \pm 1$. KobayashiNagano [6] has classified all such pairs $(\mathfrak{g}, e)$. Denote by $\mathfrak{g}_{-1}$ the $(-1)-$ eigen space of $\operatorname{ad}(e)$. We list up Lie algebras $g$ and $\operatorname{dim} g_{-1}$ for pairs $(\mathrm{g}, e)$ in the following, (Table II). 
Table I.

\begin{tabular}{|c|c|c|}
\hline $\mathfrak{g}$ & $\mathfrak{l}$ & signature of $B_{\mathfrak{p}}$ \\
\hline $\mathfrak{g} \mathfrak{I}(k, C)$ & $\mathfrak{G I}(j, C) \oplus \mathfrak{g} \mathfrak{I}(k-j, C) \oplus C$ & $(2 j(k-j), 2 j(k-j))$ \\
\hline $\operatorname{gr}(2 k, R)$ & $\mathfrak{g I}(k, C) \oplus \boldsymbol{R}$ & $(k(k-1), k(k+1))$ \\
\hline $\mathfrak{B}_{\mathrm{I}} *(2 k)$ & $\mathfrak{I}(k, C) \oplus \boldsymbol{R}$ & $(k(k+1), k(k-1))$ \\
\hline $\mathfrak{s} n(p, q)$ & $\mathfrak{z} \mathfrak{n}(i, j) \oplus \mathfrak{s} \mathfrak{u}(p-i, q-j) \oplus R$ & $\begin{array}{l}(2 i(p-i)+2 j(q-j), 2\{(p+q)(i+j) \\
\left.\left.-(i+j)^{2}-i(p-i)-j(q-j)\right\}\right)\end{array}$ \\
\hline $\mathfrak{S} \mathfrak{D}(2 k, C)$ & $\mathfrak{s} I(k, C) \oplus C$ & $(k(k-1), k(k-1))$ \\
\hline $\mathfrak{g} \mathfrak{o}(k, C)$ & $3 \mathfrak{p}(k-2, C) \oplus C$ & $(2 k-4,2 k-4)$ \\
\hline $\mathfrak{s}^{* *}(2 k)$ & $\mathfrak{z} \mathfrak{u}(j, k-j) \oplus \boldsymbol{R}$ & $(2 j(k-j), k(k-1)-2 j(k-j))$ \\
\hline $\mathrm{s}_{0} *(2 k)$ & $30 *(2 k-2) \oplus R$ & $(2 k-2,2 k-2)$ \\
\hline $\operatorname{so}(2 p, 2 q)$ & $\sin (p, q) \oplus \boldsymbol{R}$ & $\left(p^{2}+q^{2}-(p+q), 2 p q\right)$ \\
\hline $\operatorname{po}(p+2, q)$ & $\mathfrak{s} \mathfrak{b}(p, q) \oplus \boldsymbol{R}$ & $(2 p, 2 q)$ \\
\hline$w p(k, C)$ & $\mathfrak{s} \mathfrak{I}(k, C) \oplus C$ & $(k(k+1), k(k+1))$ \\
\hline $\mathfrak{g p}(k, \boldsymbol{R})$ & $\mathfrak{z} \mathfrak{u}(j, k-j) \oplus \boldsymbol{R}$ & $(2 j(k-j), k(k+1)-2 j(k-j))$ \\
\hline${ }_{p} p(p, q)$ & $\mathfrak{s} \mathfrak{u}(p, q) \oplus \boldsymbol{R}$ & $\left(p^{2}+q^{2}+(p+q), 2 p q\right)$ \\
\hline$E_{6}^{C}$ & $\mathfrak{s o}(10, C) \oplus C$ & $(32,32)$ \\
\hline$E_{6}^{2}$ & $\mathfrak{s v}^{*}(10) \oplus R$ & $(12,20)$ \\
\hline$E_{6}^{2}$ & $\mathfrak{S D}(4,6) \oplus \boldsymbol{R}$ & $(16,16)$ \\
\hline$E_{6}^{3}$ & $30(10) \oplus R$ & $(0,32)$ \\
\hline$E_{6}^{3}$ & $\mathfrak{s}(2,8) \oplus \boldsymbol{R}$ & $(16,16)$ \\
\hline$E_{6}^{3}$ & $\mathfrak{S D}^{* *}(10) \oplus R$ & $(20,12)$ \\
\hline$E_{7}^{C}$ & $E_{6}^{C} \oplus C$ & $(54,54)$ \\
\hline$E_{7}^{1}$ & $E_{6}^{2} \oplus R$ & $(24,30)$ \\
\hline$E_{7}^{2}$ & $E_{6}^{3} \oplus \boldsymbol{R}$ & $(22,32)$ \\
\hline$E_{7}^{2}$ & $E_{6}^{2} \oplus R$ & $(30,24)$ \\
\hline$E_{7}^{3}$ & $E_{6} \oplus \boldsymbol{R}$ & $(0,54)$ \\
\hline$E_{7}^{3}$ & $E_{6}^{3} \oplus \boldsymbol{R}$ & $(32,22)$ \\
\hline$E_{7}^{3}$ & $E_{6}^{4} \oplus \boldsymbol{R}$ & $(26,28)$ \\
\hline
\end{tabular}

Note: Lie algebras g are not necessarily simple.

Lemma 9.1. Let $M_{s_{c}}^{r} \subset \bar{M}_{s_{c}}^{r}(c)$ be the complete totally real parallel submanifold associated with the simple HSGLA $\mathscr{G}_{c}, c<0$. Then the following three cases are possible:

(a) The submanifold $M_{s_{\mathrm{c}}}^{r}$ is isometric to the real hyperbolic space $\mathrm{SO}(1, r) / \mathrm{SO}(r)$ of constant sectional curvature $c / 4$ and is totaly geodesic in $\bar{M}_{s_{c}}^{r}(c)$.

(b) $r=1$, i.e., $M_{y_{c}}^{1}$ is a curve in $\bar{M}_{s_{c}}^{1}(c)$.

(c) $r=0$, i.e., $M_{s_{c}}^{0}=\bar{M}_{s_{c}}^{0}(c)$ is a point. 
Table II.

\begin{tabular}{|c|c|c|c|}
\hline$g$ & $\operatorname{dim} g_{-1}$ & $\mathrm{~g}$ & $\operatorname{dim} g_{-1}$ \\
\hline $\operatorname{sI}(p+q, R)$ & $p q$ & app $(n, n)$ & $2 n^{2}+n$ \\
\hline $\operatorname{sI}(p+q, C)$ & $2 p q$ & $\ddot{z p}(n, \boldsymbol{R})$ & $n(n+1) / 2$ \\
\hline $3 \mathfrak{t} *(2 p+2 q)$ & $4 p q$ & $\mathfrak{z p}(n, C)$ & $n^{2}$ \\
\hline $\operatorname{so}(n, n)$ & $n(n-1) / 2$ & $\mathfrak{d}(n, n)$ & $2 n^{2}+n$ \\
\hline $\mathrm{s} p(2 n, C)$ & $n(n-1)$ & $E_{6}^{1}$ & 16 \\
\hline $30 *(4 n)$ & $n(2 n-1)$ & $E_{6}^{C}$ & 32 \\
\hline $\mathrm{s} p(p+1, q+1)$ & $p+q$ & $E_{6}^{4}$ & 16 \\
\hline$B d(p+q+2, C)$ & $2(p+q)$ & $E_{7}^{1}$ & 27 \\
\hline $\mathrm{s} p(n, n)$ & $n(n-1) / 2$ & $E_{7}^{C}$ & 54 \\
\hline $\mathfrak{b t}(n, n)$ & $n^{2}$ & $E_{7}^{3}$ & 27 \\
\hline
\end{tabular}

Proof. Since $\mathscr{G}_{c}$ is effective and $\mathfrak{l}=[\mathfrak{p}, \mathfrak{p}]$, the form \langle\rangle$_{\mathfrak{b}}$ is extented into a non-degenerate symmetric bilinear form \langle\rangle on $g$ which $\operatorname{ad}(T)$, $T \in \mathfrak{g}$, are skew-symmetric for. Assume that the complexification $\mathfrak{g}^{c}$ of $\mathfrak{g}$ is complex simple. Then we have $\langle\rangle=,\alpha B$ for some $\alpha \neq 0$ by Shur's lemma. Assume that $\mathrm{g}^{c}$ is not complex simple. Then the real Lie algebra $\mathrm{g}$ is isomorphic to the complexification $\mathfrak{h}^{c}$ of a compact simple Lie algebra $\mathfrak{h}$. Denote by $B_{\mathfrak{h}}$ the Killing form of $\mathfrak{h}$. Since $\operatorname{ad}(T), T \in \mathfrak{g}$, are skewsymmetric for $\langle$,$\rangle , there exist real numbers a, b$ such that

$$
\begin{aligned}
\left\langle X+\sqrt{-1} Y, X^{\prime}+\sqrt{-1} Y^{\prime}\right\rangle= & a\left\{B_{\S}\left(X, X^{\prime}\right)-B_{\natural}\left(Y, Y^{\prime}\right)\right\} \\
& +b\left\{B_{\S}\left(X, Y^{\prime}\right)+B_{\S}\left(X^{\prime}, Y\right)\right\}
\end{aligned}
$$

for $X, X^{\prime}, Y, Y^{\prime} \in \mathfrak{h}$. The involution $\rho$ commutes with a Cartan involution of $g$ (Berger [1]) and moreover the subalgebra $\mathfrak{t}$ is a complex Lie algebra for $\mathfrak{g}$ of this type contained in Table I. Hence we have $\mathfrak{p}=\mathfrak{h} \cap \mathfrak{p} \oplus \sqrt{-1} \mathfrak{h}$ $\cap \mathfrak{p}$. Since $B_{\mathfrak{q}}$ is negative definite, the signature of \langle\rangle$_{\mathfrak{p}}$ is $(\operatorname{dim} \mathfrak{h} \cap \mathfrak{p}$, $\operatorname{dim}$ $\mathfrak{h} \cap \mathfrak{p})$ by the above expression of $\langle$,$\rangle .$

Now we pick up all possible cases satisfying the conditions (1), (2), (3) by using Table I, II. Then we can see that the Lie subalgebra $\mathfrak{k}$ is isomorphic to one of the following:

$$
\mathfrak{S}_{\mathfrak{O}}(1, r) \oplus \boldsymbol{R}(r \geqq 2), \boldsymbol{C}, \boldsymbol{R} .
$$

The tangent space at $\nu$ of $\hat{M}_{s_{c}}^{r+1}$ is identified with the subspace $\mathfrak{m}=$ $\{(X, 0, X) ; X \in A\}$ of $\mathfrak{k}$. Then we note that $\mathfrak{f}=[\mathfrak{m}, \mathfrak{m}] \oplus \mathfrak{m}$. Put $p=\pi_{s_{c}}(\nu)$ $\in M_{\mathscr{g}_{c}}^{r}$ and $\tilde{\mathfrak{m}}=T_{p}\left(M_{\mathfrak{g}_{c}}^{r}\right)$. Denote by $\tilde{\mathfrak{f}}_{0}$ the holonomy algebra of curvature 
endomorphisms $R_{p}(X, Y), X, Y \in \tilde{\mathfrak{m}}$. The direct sum $\tilde{\mathfrak{f}}=\tilde{\mathfrak{f}}_{0} \oplus \widetilde{\mathfrak{m}}$ is a Lie algebra with the following product [ ]:

$$
[T, S]=T \circ S-S \circ T, \quad[T, X]=-[X, T]=T(X), \quad[X, Y]=-R_{p}(X, Y)
$$

for $T, S \in \tilde{\mathfrak{f}}_{0}, X, Y \in \widetilde{\mathfrak{m}}$. Then we can easily see that $[\mathrm{m}, \mathrm{m}] \oplus \mathfrak{m}$ is isomorphic to $\tilde{\mathfrak{l}} \oplus \boldsymbol{R}$ and thus $\mathfrak{\mathfrak { l }}$ is isomorphic to $\tilde{\mathfrak{l}} \oplus \boldsymbol{R}$.

Assume that $\mathfrak{f}$ is isomorphic to $\mathfrak{g} \mathfrak{0}(1, r) \oplus \boldsymbol{R}$. Then $\tilde{\mathfrak{E}}$ is isomorphic to $\mathfrak{g} \mathfrak{p}(1, r)$. Hence $M_{\mathfrak{s}_{c}}^{r}$ is locally isometric to the riemannian symmetric space $\mathrm{SO}(1, r) / \mathrm{SO}(r)$ by Proposition 4.2. Then the submanifold $M_{s_{c}}^{r}$ is totally geodesic in $\bar{M}_{s_{c}}^{r}(c)$ (See the proof of Proposition 4.2). This implies that $M_{\mathscr{s}_{c}}^{r}$ is globally isometric to the real hyperbolic space of constant sectional curvature $c / 4$.

Assume that $\mathfrak{f}$ is isomorphic to either $\boldsymbol{C}$ or $\boldsymbol{R}$. Then we have $\operatorname{dim}$ $\hat{M}_{\mathfrak{s}_{c}}^{r+1}=1,2$ and thus $r=0,1$.

q.e.d.

Now we study OJA's $\mathscr{A}_{c}$ and HSGLA's $\mathscr{G}_{c}$ for cases (a), (b), (c) respectively.

Case (a). Assume that $M_{\mathscr{s}_{c}}^{r}$ is totally geodesic in $\bar{M}_{\mathscr{s}_{c}}^{r}(c)$. Let $A=\{E\}_{R}$ $\oplus H$ be the orthogonal decomposition of $A$ for \langle\rangle . Identify the tangent space $T_{\nu}\left(\hat{M}_{\mathfrak{F}_{c}}^{r+1}\right)$ with the subspace $\left(\mathfrak{g}_{-1} \oplus \mathfrak{g}_{1}\right) \cap \mathfrak{p}$ of $\mathfrak{p}$. Then, since $J_{\mathfrak{p}}(\nu)=$ $(E, 0,-E)$, the horizontal space $H_{\nu}\left(\hat{M}_{\mathfrak{s}_{c}}^{r+1}\right)$, the vertical space $V_{\nu}\left(\hat{M}_{\mathfrak{s}_{c}}^{r+1}\right)$ at $\nu \in \hat{M}_{\mathscr{s}_{c}}^{r+1}$ are given by

$$
H_{\nu}\left(\hat{M}_{\mathfrak{s}_{c}}^{r+1}\right)=\{(X, 0,-X) ; X \in H\}, \quad V_{\nu}\left(\hat{M}_{\mathscr{s}_{c}}^{r+1}\right)=\{(E, 0,-E)\}_{R}
$$

respectively. Denote by $\sigma$ the second fundamental form at $\nu$ of the submanifold $\hat{M}_{\mathfrak{s}_{c}}^{r+1}$ in the pseudo-Hermitian space $F_{s_{c}}^{r+1}=\left(\mathfrak{p}, J_{\mathfrak{p}},\langle\rangle_{p}\right)$. Then, since $M_{\mathscr{G}_{c}}^{r}$ is totally geodesic in $\bar{M}_{\mathscr{S}_{c}}^{r}(c)$, we have

$$
\sigma((X, 0,-X),(Y, 0,-Y))=-(c / 4)\langle X, Y\rangle_{\nu}
$$

for $X, Y \in H$ by (4.1), (4.6). On the other hand, the second fundamental form $\sigma$ is also given by

$$
\sigma((X, 0,-X),(Y, 0,-Y))=\left(0, T_{X \cdot Y}, 0\right)
$$

for $X, Y \in A$ (See the proof of Theorem 6.3, (1)). Hence we have $T_{X \cdot Y}=$ $(c / 4)\langle X, Y\rangle T_{E}$ for $X, Y \in H$. This implies that $X \cdot Y=(c / 4)\langle X, Y\rangle E$ for $X, Y \in H$.

Fix an integer $r \geqq 1$ and a negative number $c$. Let $\mathscr{P}=(P,\langle\rangle)$ be an object of $r$-dimensional real vector space $P$ and positive definite inner 
product $\left\langle>\right.$ on $P$. And let $E$ be an element. Then put $A=\{E\}_{\boldsymbol{R}} \oplus P$ and define a product on $A$ by

$$
X \cdot Y=(c / 4)\langle X, Y\rangle E, \quad Z \cdot E=E \cdot Z=Z
$$

for $X, Y \in P, Z \in A$ and moreover extend the inner product $\langle>$ on $P$ into a non-degenerate symmetric bilinear form $\langle>$ on $A$ by

$$
\langle E, P\rangle=\{0\}, \quad\langle E, E\rangle=4 / c .
$$

Then the object $\mathscr{A}(r, c, \mathscr{P}, E)=(A,\langle\rangle)$ is an OJA with the unity $E$ satisfying the conditions $\left(\mathrm{E}_{c} 1\right),\left(\mathrm{E}_{c} 2\right)$. Moreover such two OJA's $\mathscr{A}(r, c$, $\mathscr{P}, E), \mathscr{A}\left(r^{\prime}, c^{\prime}, \mathscr{P}^{\prime}, E^{\prime}\right)$ are equivalent to each other if and only if $r=r^{\prime}$ and $c=c^{\prime}$. Our OJA $\mathscr{A}_{c}$ is equivalent to $\mathscr{A}(r, c, \mathscr{H}, E)$, where $r=\operatorname{dim}$ $A-1, \mathscr{H}=\left(H,\left\langle>\left.\right|_{H}\right)\right.$.

Denote by $\mathscr{G}(r, c, \mathscr{H}, E)$ the HSGLA coming from the OJA $\mathscr{A}(r, c, \mathscr{H}, E)$. Then $\mathscr{G}_{c}$ is equivalent to $\mathscr{G}(r, c, \mathscr{H}, E)$. We show that the Lie algebra $\mathfrak{g}$ underlying $\mathscr{G}(r, c, \mathscr{H}, E)$ is isomorphic to $\mathfrak{B}(r+2,1)$. Let $\left\{e_{1}, \cdots, e_{r}\right\}$ be an orthonormal basis of $H$ and put $e_{0}=(\sqrt{-c} / 2) E$. Then $\left\{e_{0}, e_{1}, \cdots, e_{r}\right\}$ is an orthonormal basis of $A$. Define a matrix $\alpha(F)=\left(\alpha(F)_{i j}\right) \in M_{r+1}(R)$ for $F \in L$ by $F\left(e_{i}\right)=\sum_{j} \alpha(F)_{i j} e_{j}, 0 \leqq i \leqq r$, and put

$$
\beta(F)=\alpha(F)+\left\langle F\left(e_{0}\right), e_{0}\right\rangle 1_{r+1}
$$

where $1_{r+1}$ denotes the unit matrix of degree $r+1$. Then we can see that $\beta(F) \in \mathfrak{S} \mathfrak{D}(r+1)$ by (9.2). Put

$$
\mathscr{X}(X)=\left(\begin{array}{c}
x_{0} \\
\vdots \\
\vdots \\
x_{r}
\end{array}\right), \quad \overline{\mathscr{X}(X)}=\left(\begin{array}{c}
x_{0} \\
-x_{1} \\
\vdots \\
-x_{r}
\end{array}\right)
$$

for $X=\sum_{j} x_{j} e_{j} \in A$. Define a linear mapping $\Phi$ of $g$ onto $\mathfrak{s}_{\mathfrak{j}}(r+2,1)$ by

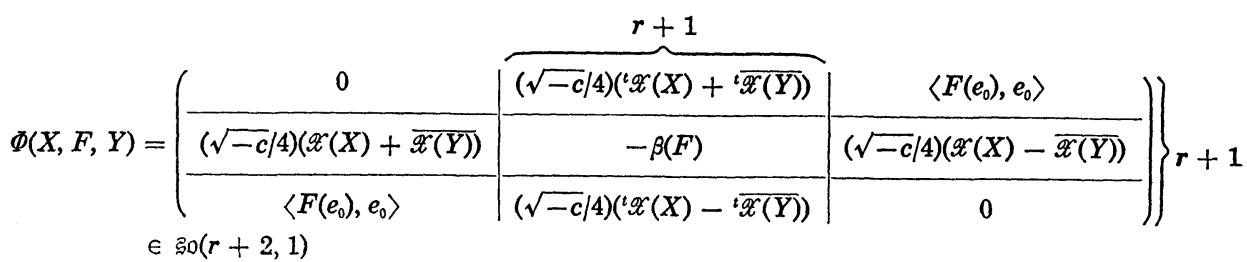

for $(X, F, Y) \in \mathfrak{g}$. Then we can see that $\Phi$ is a Lie algebra isomorphism by (9.2). Hence $\mathfrak{g}$ is isomorphic to $\mathfrak{g} 0(r+2,1)$. 
Various objects underlying the HSGLA $\mathscr{G}(r, c, \mathscr{H}, E)$ may be expressed explicitly through the isomorphism $\Phi$.

Case (b). Assume that $r=1$, i.e., $M_{\mathfrak{g}_{c}}^{1}$ is a curve in $\bar{M}_{\mathfrak{s}_{c}}^{1}(c)$. Since the curve $M_{\mathfrak{y}_{c}}^{1}$ is parallel in $\bar{M}_{\mathscr{s}_{c}}^{1}(c)$, it is a Frenet curve of osculating rank 0 or 1 . Denote by $\kappa=\kappa_{1} \geqq 0$ the Frenet curvature of $M_{\mathscr{s}_{c}}^{1}$ and by $\sigma_{M}$ the second fundamental form of $M_{\mathscr{g}_{c}}^{1} \subset \bar{M}_{\vartheta_{c}}^{1}(\mathrm{c})$. Then, since $M_{\mathfrak{s}_{c}}^{1}$ is totally real, we have

$$
\sigma_{M}(X, X)=\kappa|X| J_{\bar{M}}(X)
$$

for a tangent vector field $X$ of $M_{\mho_{c}}^{1}$, where $J_{\bar{M}}$ denotes the complex structure of $\bar{M}_{\mathscr{s}_{c}}^{1}(c)$ and $|*|$ denotes the length of $*$. Let $A=\{E\}_{\boldsymbol{R}} \oplus H$ be the orthogonal decomposition of $A$ for \langle\rangle . Then the horizontal space $H_{\nu}\left(\hat{M}_{s_{c}}^{2}\right)$, the vertical space $V_{\nu}\left(\hat{M}_{\mathscr{s}_{c}}^{2}\right)$ at $\nu$ are given as in the case (a). Denote by $\sigma$ the second fundamental form at $\boldsymbol{\nu}$ of $\hat{M}_{\mathscr{S}_{c}}^{2} \subset F_{\mathscr{s}_{c}}^{2}$. Then we have $\sigma(X, X)=\left(0, \kappa|X| T_{X}+(c / 4)\langle X, X\rangle \mathrm{id}, 0\right)$ for $X \in H$ by (4.1), (4.6), (9.4). Hence we have $T_{X \cdot X}=\kappa|X| T_{X}+(c / 4)\langle X, X\rangle T_{E}$ for $X \in H$ by (9.1). This implies that $X \cdot X=\kappa|X| X+(c / 4)\langle X, X\rangle E$ for $X \in H$.

Fix a negative number $c$ and a non-negative number $\kappa$. Let $\mathscr{P}=$ $(P,\langle\rangle)$ be an object of 1 -dimensional real vector space $P$ and positive definite inner product $\left\langle>\right.$ on $P$. Then put $A=\{E\}_{R} \oplus P$ and define a product - on $A$ by

$$
X \cdot X=\kappa|X| X+(c / 4)\langle X, X\rangle E, \quad Y \cdot E=E \cdot Y=Y
$$

for $X \in P, Y \in A$, and moreover extend the inner product $\langle>$ on $P$ into a non-degenerate symmetric bilinear form $\langle>$ on $A$ by

$$
\langle E, P\rangle=\{0\}, \quad\langle E, E\rangle=4 / c .
$$

Then the object $\mathscr{A}(c, \kappa, \mathscr{P}, E)=(A,\langle\rangle)$ is an OJA with the unity $E$ satisfying the conditions $\left(\mathrm{E}_{c} 1\right),\left(\mathrm{E}_{c} 2\right)$. Moreover such two OJA's $\mathscr{A}(c, \kappa$, $\mathscr{P}, E), \mathscr{A}\left(c^{\prime}, \kappa^{\prime}, \mathscr{P}^{\prime}, E^{\prime}\right)$ are equivalent to each other if and only if $c=c^{\prime}$ and $\kappa=\kappa^{\prime}$. Our OJA $\mathscr{A}_{c}$ is equivalent to $\mathscr{A}(c, \kappa, \mathscr{H}, E)$, where $\kappa$ denotes the Frenet curvature of $M_{\mathscr{s}_{c}}^{1}$ and $\mathscr{H}=\left(H,\left\langle>\left.\right|_{H}\right)\right.$.

Denote by $\mathscr{G}(c, \kappa, \mathscr{H}, E)$ the HSGLA coming from $\mathscr{A}(c, \kappa, \mathscr{H}, E)$ and by $g$ the Lie algebra underlying $\mathscr{G}(c, \kappa, \mathscr{H}, E)$. Let $e_{1}$ be a unit vector of $H$ and put $e_{0}=(\sqrt{-c} / 2) E$. Then $\left\{e_{0}, e_{1}\right\}$ is an orthonormal basis of $A$. The matrix representation $D(\eta)$ of $T_{\eta}$ for this basis is given by 


$$
D(\eta)=\left(\begin{array}{cc}
c / 4 & \sqrt{-c} \kappa / 4 \\
-\sqrt{-c} \kappa / 4, & c / 4+\kappa^{2} / 2
\end{array}\right)
$$

by (9.5), (9.6). Then we consider separately the following three cases (i), (ii), (iii).

(i) $\kappa=\sqrt{-c}$ : In this case the eigen values of $D(\eta)$ are zero and thus $T_{\eta}$ is nilpotent. This implies that the HSGLA $\mathscr{G}(c, \sqrt{-c}, \mathscr{H}, E)$ is equivalent to the almost nilpotent HSGLA $\mathscr{G}(2, c,\{0\}, E, \eta)$.

(ii) $\kappa>\sqrt{-c}$ : In this case the matrix $D(\eta)$ has two distinct nonzero real eigen values $\lambda_{i}, i=1,2$. Let $A_{i}, i=1,2$, be eigen spaces of $T_{\eta}$ for eigen values $\lambda_{i}$ respectively. Since $A$ is spanned over $R$ by the vectors $E$, $\eta$, the subspaces $A_{i}$ are ideals of $A$ by (9.5), and thus $A$ is decomposed into the sum $A_{1} \oplus A_{2}$ of ideals $A_{i}$. Since $T_{\eta}$ is non-degenerate on $A$, the decomposition of $A$ induces a decomposition $\mathscr{A}_{1} \oplus \mathscr{A}_{2}$ of the OJA $\mathscr{A}(c, \kappa$, $\mathscr{H}, E)$ by Lemma 7.2. Hence the HSGLA $\mathscr{G}(c, \kappa, \mathscr{H}, E)$ is decomposed into the sum $\mathscr{G}_{1} \oplus \mathscr{G}_{2}$ of two simple HSGLA's $\mathscr{G}_{i}$. These HSGLA's $\mathscr{G}_{i}$ will be studied in the case (c).

(iii) $0 \leqq \kappa<\sqrt{-c}$ : Define a complex structure $j_{\kappa}$ on $A$ by

$$
\left\{\begin{array}{l}
j_{\kappa}\left(e_{0}\right)=\left(1 / \sqrt{-c-\kappa^{2}}\right)\left(-\kappa e_{0}+\sqrt{-c} e_{1}\right), \\
j_{\kappa}\left(e_{1}\right)=\left(1 / \sqrt{-c-\kappa^{2}}\right)\left(-\sqrt{-c} e_{0}+\kappa e_{1}\right),
\end{array}\right.
$$

and denote by $C_{k}$ the 1-dimensional complex linear space $\left(A, j_{k}\right)$. Moreover identify $\boldsymbol{C}_{\boldsymbol{c}}$ with the complex space $\boldsymbol{C}$ by

$$
C_{\kappa} \ni x e_{0}+y j_{\kappa}\left(e_{0}\right) \longleftrightarrow(\sqrt{-c} / 2)(x+y i) \in C .
$$

Then the Jordan product - on $\boldsymbol{C}_{\boldsymbol{x}}$ corresponds with the canonical product . on $C$ under this identification by (9.5). Hence the $R$-linear endomorphisms $F \in L$ are $C$-linear endomorphisms on $C$. Let $\alpha(F)$ be a complex number such that $F(Z)=\alpha(F) \cdot Z$ for $Z \in C$. Define a linear mapping $\Phi_{\kappa}$ of $g$ onto $\mathfrak{g l}(2, C)$ by

$$
\Phi_{\star}((Z, F, W))=\left(\begin{array}{cc}
-\alpha(F) / 2 & -W / 2 \\
Z / 2 & \alpha(F) / 2
\end{array}\right)
$$

for $(Z, F, W) \in g$. Then we can easily see that $\Phi_{\kappa}$ is a Lie algebra isomorphism. Hence $\mathrm{g}$ is isomorphic to $\mathfrak{g}(2, C)$.

Various objects underlying $\mathscr{G}(c, \kappa, \mathscr{H}, E)$ may be expressed explicitly through the isomorphism $\Phi_{x}$. 
Finally we note that $\mathscr{G}(c, 0, \mathscr{H}, E)$ is equivalent to the HSGLA $\mathscr{G}(1, c$, $\mathscr{P}, E)$ in the case (a).

Case (c). Assume that $r=0$. Then we have $A=\{E\}_{R}$ and $\langle E, E\rangle$ $=4 / c$. Let $\mathscr{A}_{-c}=(A,-\langle\rangle)$ and $\mathscr{G}_{-c}=\left(\mathfrak{g}=\sum g_{\mu}, \rho, J_{p},-\langle\rangle_{p}\right)$. Then $\mathscr{A}_{-c}$ (resp. $\mathscr{G}_{-c}$ ) is equivalent to the OJA (resp. HSGLA) corresponding to the object $\left(D^{1},-c\right)$, where $D^{1}$ denotes the 1-dimensional symmetric bounded domain of tube type. Particularly, $g$ is isomorphic to $\mathfrak{g}(2, R)$. This OJA $\mathscr{A}_{c}$ (resp. HSGLA $\mathscr{G}_{c}$ ) will be denoted by $\mathscr{A}(c, E)$ (resp. $\mathscr{G}(c, E)$ ).

Summing up the above cases, we have the following

TheOREm 9.2. Let $\mathscr{G}_{c}$ be the HSGLA coming from an OJA $\mathscr{A}_{c}$ with the unity $E$ satisfying the conditions $\left(\mathrm{E}_{c} 1\right),\left(\mathrm{E}_{c} 2\right)$. Assume that $c<0$ and $\mathscr{G}_{c}$ is simple. Then $\mathscr{G}_{\mathrm{c}}$ is equivalent to one of the following HSGLA's:

$\mathscr{G}(r, c, \mathscr{P}, E)(r \geqq 1)$ in Case (a), $\mathscr{G}(c, \kappa, \mathscr{P}, E)(0<\kappa<\sqrt{-c})$ in Case (b), (iii), and $\mathscr{G}(c, E)$ in Case (c).

\section{REFERENCES}

[1] M. Berger, Espaces symétriques non compacts, Ann. Sci. École Norm. Sup. (3), 74. (1957), 85-177.

[2] M. Cahen and M. Parker, Pseudo-riemannian symmetric spaces, Memo. of the Amer. Math. Soc., No. 229, 24 (1980).

[3] D. Ferus, Symmetric submanifolds of euclidean space, Math. Ann., 247 (1980), 81-93.

[ 4 ] - Immersionen mit paralleler zweiter Fundamentalform: Beispiele und NichtBeispiele, Manuscripta Math., 12 (1974), 153-162.

[5] S. Helgason, Differential geometry, Lie groups and Symmetric spaces, Academic Press, New York, 1978.

[6] S. Kobayashi and T. Nagano, On filtered Lie algebras and geometric structures I, J. Math. and Mech., 13 (1964), 875-907.

[ 7 ] S. Kobayashi and K. Nomizu, Foundations of Differential geometry I, II, Wiley (Interscience), 1963 and 1969.

[ 8 ] H. Naitoh, Totally real parallel submanifolds in $P^{n}(c)$, Tokyo J. Math., 4, No. 2 (1981), 279-306.

[ 9 ] —_, Parallel submanifolds of complex space forms I, Nagoya Math. J., 90 (1983), 85-117.

[10] H. Naitoh and M. Takeuchi, Totally real submanifolds and symmetric bounded domains, Osaka J. Math., 19 (1982), 717-731.

[11] I. Satake, Algebraic structures of symmetric domains, Iwanami Shoten, Publishers and Princeton Univ. Press, 1981.

[12] M. Takeuchi, Parallel submanifolds of space forms. In: Manifolds and Lie groups, in honor of Y. Matsushima, 429-447. Progress in Math., Vol. 14, Birkhäuser, 1981.

\section{Department of Mathematics}

Yamaguchi University

Yamaguchi 753, Japan 